\title{
THE LOW-FREQUENCY THEORY OF ELASTIC WAVE SCATTERING*
}

\author{
BY \\ GEORGE DASSIOS (University of Patras) \\ AND \\ KIRIAKIE KIRIAKI ( National Technical University of Athens)
}

\begin{abstract}
An incident longitudinal, or transverse, plane wave is scattered by a bounded region immersed in an infinite isotropic and homogeneous elastic medium. The region could be either a rigid scatterer or a cavity. Integral representations for the total displacement field, as well as for the introduced spherical scattering amplitudes are given explicitely in a compact form. Representations for the scattering cross-section whenever the incident wave is a longitudinal or a transverse wave are also provided. Using Papkovich potentials and low-frequency techniques the scattering problems are reduced to an iterative sequence of potential problems which can be solved successively in terms of expansions in appropriate harmonic functions. In each one of the four cases (longitudinal and transverse incidence on rigid scatterer and cavity) the corresponding exterior boundary value problems that specify the approximations as well as the analytic expressions for the scattering amplitudes and the scattering cross-section are given explicitly. The leading low-frequency term of the scattering cross-section for a rigid scatterer is independent of the wave number while for the case of a cavity it is proportional to the fourth power of the wave number. The low-frequency limit of the displacement field which corresponds to the static problem when the scatterer is a cavity, does not depend on the geometrical characteristics of the scatterer and it is always a constant.
\end{abstract}

1. Introduction. The problem of scattering of a plane harmonic elastic wave by an obstacle appears as an exterior boundary value problem for the time independent Navier equation with specific boundary conditions on the surface of the obstacle and prescribed asymptotic form (known as radiation conditions) in the neighborhood of infinity.

The usual boundary conditions correspond to a rigid scatterer, described by the vanishing of the total displacement field on the surface of the obstacle, to a cavity, which is described by the vanishing of the surface traction and to a penetrable body, on the surface of which both the displacement and the traction fields are continuous. The

${ }^{*}$ Received by the editors June 8,1983 . This work constitutes a part of the doctoral dissertation of the second author performed under the guidance of the first. 
radiation conditions, which are the same for any type of boundary conditions, impose the requirement that the scattered wave propagates away from the scatterer and it is diminished as the inverse first power of the distance from the scatterer.

The incident wave is a time harmonic plane wave propagating along a direction $\hat{\mathbf{k}}$ and polarized either in the direction of propagation $\hat{\mathbf{k}}$ (longitudinal wave), or perpendicular to the propagation vector $\hat{\mathbf{k}}$ (transverse wave). In either case the incident wave has no singularities in the finite Euclidean space. The first attempt to solve the scattering problem for the simplest three dimensional geometry, that of the sphere is due to Ying and Truell [19] who, in 1956, solved the problem of scattering of a plane longitudinal wave by a rigid sphere, a spherical cavity and a penetrable sphere. Four years later Einspruch, Witterholt and Truell [9] succeeded in solving the same problem with an incident transverse plane wave. The difficulty caused by a transverse incident wave, over the simpler case of a longitudinal incident wave, is due to the polarization vector which destroys the azimouthal symmetry of the problem.

The general theory of scattering of elastic waves is very well exposed by Kupradze [10] who discusses many interesting quantitative as well as qualitative aspects of elastic wave propagation and scattering, including the specific expressions for the fundamental solutions, the radiation conditions and a few integral representations.

The present work is very much affected by Kupradze's book.

The work of Barratt and Collins [2] provides the first investigation of the scattering amplitude as well as the scattering cross-section. On the basis of their formulae they have evaluated the scattering cross-section for a sphere and a cylinder at the low-frequency approximation. Barratt and Collins are based on Kupradze's work too.

Using the work of Barratt and Collins, Lawrence [11] has calculated the scattering cross-section for a rigid scatterer which has three mutually perpendicular planes of symmetry and the direction of incidence coincides with one of the axis of symmetry. His work refers also to the low-frequency case. In a latter paper Lawrence [12] improved his previous result by letting the direction of incidence have any orientation with respect to the symmetry axis of the scatterer. As an application of his technique he found the low-frequency scattering cross-section of a rigid ellipsoid.

Integral representations for the displacement field are also given by Banauch [1], Wheeler and Sternberg [18] who proved uniqueness theorems as well and Pao and Varatharajulu [13]. Waterman [16, 17] has given a matrix formulation for scattering of elastic waves. For low-frequency scattering and corresponding integral theorems one can use Twersky's work [14, 15].

In this work an integrated and systematic theory for scattering of elastic waves by convex obstacles is developed. The incident wave could be either a longitudinal or a transverse plane wave and the scatterer is a rigid body or a cavity. Integral representations for the total (incident plus scattered) field are given which involve the surface stress operator on the surface of the scatterer. Spherical scattering amplitudes are introduced through integral representations of the asymptotic form of the total field, in such a way that the boundary conditions can be directly inserted in the representations. The evaluation of the spherical scattering amplitudes demand the calculation of exactly one type of 
integral for each one of the two cases, for the rigid scatterer and the cavity. Similar representations for the scattering amplitude, both for longitudinal and transverse incident waves, are established. To the leading order approximation, in the low-frequency region, the scattering cross-section for the rigid scatterer is independent of the wave number, while for the cavity it is proportional to the fourth power of the wave number.

The four scattering problems, corresponding to the combinations of the two types of incident waves with the two types of boundary conditions, are studied in the case of the low-frequency expansions where the characteristic dimension $\alpha$ of the obstacle is much smaller than the wavelength of the incident wave. The value of $\alpha$ is defined as the radius of the smallest sphere that circumscribes the obstacle.

All the wave fields of the problem as well as the fundamental dyadic solution are expanded in power series of the wave number. Using these expansions the wave problem is reduced to a sequence of potential problems, which by means of Papkovich potential representations for the displacement field can be solved iteratively by means of appropriate harmonic functions. It is known that when no body forces are present the vector and the scalar Papkovich potentials are no longer independent and therefore only the vector potential is needed to describe the problem. Nevertheless if both potentials are introduced their dependence can be used to overcome technical difficulties caused by nontrivial coordinate systems.

The particular potential problems that determine all the coefficients are stated explicitely. Integral representations for every coefficient, as well as their asymptotic form, far away from the scatterer, are found. The far field form provides particular solutions of the corresponding Poisson equation. To a large extent the analysis of the potential problems for the successive coefficients is guided by the relative work of the first author $[3,4,5,6]$ for the scattering of sound waves.

Following Twersky's ideas it is succeeded to show that the leading term approximation of the scattering cross-section can be obtained by using less low-frequency coefficients than those obtained by Barratt-Collins [2]. It is also showed that the lowest approximation of the displacement field, for the case of a cavity is a constant, independent of the shape of the scatterer.

2. Formulation of the problem. Let $V^{-}$be a bounded, convex and closed subset of $R^{3}$, having a smooth boundary $S$. The set $V^{-}$will be referred to as the scatterer. Let $V$ be the complement of $V^{-}$. Assume that the space $V$ is filled with an isotropic and homogeneous elastic medium specified by the Lamé constants $\lambda$ and $\mu$. If the harmonic time dependence $e^{-i \omega t}$ is suppressed, then the displacement field $\mathbf{u}(\mathbf{r})$ satisfies the time-independent linearized equation of dynamic elasticity.

$$
\mu \Delta \mathbf{u}+(\lambda+\mu) \nabla(\nabla \cdot \mathbf{u})+\rho \omega^{2} \mathbf{u}=0
$$

where it is assumed that there are no body forces, $\rho$ is the mass density and $\omega$ is the angular frequency. Eq. (1) is also written as

$$
c_{p}^{2} \nabla(\nabla \cdot \mathbf{u})-c_{s}^{2} \nabla \times(\nabla \times \mathbf{u})+\omega^{2} \mathbf{u}=\mathbf{0}
$$


where

$$
c_{s}^{2}=\mu / \rho
$$

and

$$
c_{p}^{2}=(\lambda+2 \mu) / \rho
$$

when the vector identity

$$
\nabla \times(\nabla \times \mathbf{u})=\nabla(\nabla \cdot \mathbf{u})-\Delta \mathbf{u}
$$

is used. By scaling the space variables it is possible to have $\rho=1$ and this is done in the rest of this work. It is well known that the solution of (2) is the sum of a longitudinal wave $\mathbf{u}^{p}$ propagating with the phase velocity $c_{p}$ and a transverse wave $\mathbf{u}^{s}$ propagating with the phase velocity $c_{s}$, i.e.,

$$
\mathbf{u}(\mathbf{r})=\mathbf{u}^{p}(\mathbf{r})+\mathbf{u}^{s}(\mathbf{r})
$$

where $\mathbf{u}^{p}$ is usually called the $P$-wave and $\mathbf{u}^{s}$ the $S$-wave.

Each one of the waves $\mathbf{u}^{p}$ and $\mathbf{u}^{s}$ satisfies the wave equation with phase velocities $c_{p}$ and $c_{s}$ respectively. Both $\mathbf{u}^{p}$ and $\mathbf{u}^{s}$ waves have the same angular frequency $\omega$ which is related to the phase velocities $c_{p}$ and $c_{s}$ by the relations

$$
\omega=c_{p} k_{p}=c_{s} k_{s},
$$

where $k_{p}=2 \pi / \lambda_{p}$ and $k_{s}=2 \pi / \lambda_{s}$ are the wave numbers of the $P$ and the $S$-waves respectively, while $\lambda_{p}$ and $\lambda_{s}$ are the relative wave lengths. Consider, an incident longitudinal plane wave

$$
\boldsymbol{\Phi}^{p}(\mathbf{r})=\hat{\mathbf{k}} e^{i k_{p} \hat{\mathbf{k}} \cdot \mathbf{r}}
$$

where $\hat{\mathbf{k}}\left({ }^{1}\right)$ is the unit propagation vector, and an incident transverse plane wave

$$
\boldsymbol{\Phi}^{s}(\mathbf{r})=\hat{\mathbf{b}} e^{i k_{s} \hat{\mathbf{k}} \cdot \mathbf{r}}
$$

where the polarization vector $\hat{\mathbf{b}}$ is perpendicular to the direction of propagation $\hat{\mathbf{k}}$, i.e.,

$$
\hat{\mathbf{b}} \cdot \hat{\mathbf{k}}=0 \text {. }
$$

The harmonic time dependence $e^{-i \omega t}$ has also been eliminated from $\boldsymbol{\Phi}^{p}$ and $\boldsymbol{\Phi}^{s}$. The scatterer $V^{-}$can be either a rigid scatterer, in which case

$$
\boldsymbol{\Phi}(\mathbf{r})+\mathbf{u}(\mathbf{r})=\mathbf{0}, \quad \mathbf{r} \in S,
$$

or a cavity, in which case

$$
T(\mathbf{\Phi}(\mathbf{r})+u(\mathbf{r}))=\mathbf{0}, \quad \mathbf{r} \in S
$$

where $\boldsymbol{\Phi}$ is either $\boldsymbol{\Phi}^{p}$ or $\boldsymbol{\Phi}^{s}$,

$$
T=2 \mu \hat{\mathbf{n}} \cdot \nabla+\lambda \hat{\mathbf{n}} \operatorname{div}+\mu \hat{\mathbf{n}} \times \operatorname{rot}
$$

is the surface stress operator and $\hat{\mathbf{n}}$ is the exterior unit normal on $S$. The scattering problems considered in the present work are stated as follows. An incident longitudinal, or

\footnotetext{
(')The hat " " on the top of a vector indicates that the vector has unit magnitude.
} 
transverse, plane wave $\boldsymbol{\Phi}$ is scattered by a rigid inclusion, or a cavity. As a result of the scattering process a scattered wave $\mathbf{u}$ emanates from the scatterer. In the elastic medium $V$ the total displacement field is the superposition of the incident and the scattered field

$$
\boldsymbol{\Psi}(\mathbf{r})=\boldsymbol{\Phi}(\mathbf{r})+\mathbf{u}(\mathbf{r}), \quad \mathbf{r} \in S
$$

The longitudinal part $\mathbf{u}^{p}$ of the scattered field $\mathbf{u}$ satisfies the radiation conditions

$$
\lim _{r \rightarrow \infty} \mathbf{u}^{p}(\mathbf{r})=\mathbf{0}, \quad \lim _{r \rightarrow \infty} r\left(\frac{\partial \mathbf{u}^{p}(\mathbf{r})}{\partial r}-i k_{p} \mathbf{u}^{p}(\mathbf{r})\right)=\mathbf{0},
$$

uniformly over directions. The transverse part $\mathbf{u}^{s}$ of $\mathbf{u}$ satisfies the radiation conditions

$$
\lim _{r \rightarrow \infty} \mathbf{u}^{s}(\mathbf{r})=\mathbf{0}, \quad \lim _{r \rightarrow \infty} r\left(\frac{\partial \mathbf{u}^{s}(\mathbf{r})}{\partial r}-i k_{s} \mathbf{u}^{s}(\mathbf{r})\right)=\mathbf{0},
$$

uniformly over directions. The above form (15) and (16) of the radiation conditions are due to Kupradze [10]. The scattering problem consists in finding the field $\Psi$ that satisfies the time independent Navier equation (1), or (2) in $V$, the boundary condition (11) or (12) on $S$, while u satisfies the radiation conditions (15) and (16). These are actually four scattering problems corresponding to the combinations of the two types of incident waves with the two types of boundary conditions.

3. Integral representations. Betti's third formula [10] for the vector fields $\mathbf{u}$ and $\boldsymbol{\nu}$ which have continuous second order derivatives, states that

$$
\begin{aligned}
\int_{\Omega}\left[\mathbf{u}\left(\mathbf{r}^{\prime}\right) \cdot \Delta^{*} \boldsymbol{\nu}\left(\mathbf{r}^{\prime}\right)-\right. & \left.\boldsymbol{\nu}\left(\mathbf{r}^{\prime}\right) \cdot \Delta^{*} \mathbf{u}\left(\mathbf{r}^{\prime}\right)\right] d V \\
& =\int_{\partial \Omega}\left[\mathbf{u}\left(\mathbf{r}^{\prime}\right) \cdot T \boldsymbol{\nu}\left(\mathbf{r}^{\prime}\right)-\boldsymbol{\nu}\left(r^{\prime}\right) \cdot T \mathbf{u}\left(\mathbf{r}^{\prime}\right)\right] d s
\end{aligned}
$$

where

$$
\Delta^{*}=c_{s}^{2} \Delta+\left(c_{p}^{2}-c_{s}^{2}\right) \nabla \nabla .
$$

$T$ is the surface stress operator given by (13) and $\Omega$ is a bounded regular (in the sense that Gauss Theorem can be applied) domain. Let $\tilde{\Gamma}\left(\mathbf{r}, \mathbf{r}^{\prime}\right)\left({ }^{2}\right)$ be the fundamental dyadic solution of equation (1), i.e.

$$
\left(\Delta_{\mathbf{r}^{\prime}}^{*}+\omega^{2}\right) \tilde{\Gamma}\left(\mathbf{r}, \mathbf{r}^{\prime}\right)=-4 \pi \delta\left(\mathbf{r}-\mathbf{r}^{\prime}\right) \tilde{\mathbf{I}},
$$

where: $\tilde{\mathbf{I}}=\hat{\mathbf{x}}_{1} \otimes \hat{\mathbf{x}}_{1}+\hat{\mathbf{x}}_{2} \otimes \hat{\mathbf{x}}_{2}+\hat{\mathbf{x}}_{3} \otimes \hat{\mathbf{x}}_{3}$ is the identity dyadic and $\delta\left(\mathbf{r}-\mathbf{r}^{\prime}\right)$ represents the Dirac measure concentrated at $\mathbf{r}$. Then

$$
\tilde{\Gamma}\left(\mathbf{r}, \mathbf{r}^{\prime}\right)=\sum_{k, j=1}^{3} \Gamma_{k j} \hat{\mathbf{x}}_{k} \otimes \hat{\mathbf{x}}_{j}
$$

$\left({ }^{2}\right)$ The symbol " " on the top of a capital letter denotes a dyadic (second rank tensor). 
and

$$
\begin{aligned}
\Gamma_{k j}\left(\mathbf{r}, \mathbf{r}^{\prime}\right)= & \frac{k_{s}^{2}}{\omega^{2}} \frac{e^{i k_{s}\left|\mathbf{r}-\mathbf{r}^{\prime}\right|}}{\left|\mathbf{r}-\mathbf{r}^{\prime}\right|} \delta_{k j}-\frac{1}{\omega^{2}} \frac{\partial^{2}}{\partial x_{k} \partial x_{j}}\left[\frac{e^{i k_{p}\left|\mathbf{r}-r^{\prime}\right|}}{\left|\mathbf{r}-\mathbf{r}^{\prime}\right|}-\frac{e^{i k_{s}\left|\mathbf{r}-\mathbf{r}^{\prime}\right|}}{\left|\mathbf{r}-\mathbf{r}^{\prime}\right|}\right] \\
= & \frac{k_{s}^{2}}{\omega^{2}} \frac{e^{i k_{s}\left|\mathbf{r}-\mathbf{r}^{\prime}\right|}}{\left|\mathbf{r}-\mathbf{r}^{\prime}\right|} \delta_{k j}+\frac{e^{i k_{p}\left|\mathbf{r}-\mathbf{r}^{\prime}\right|}\left(x_{k}-x_{k}^{\prime}\right)\left(x_{j}-x_{j}^{\prime}\right)}{\omega^{2}\left|\mathbf{r}-\mathbf{r}^{\prime}\right|^{3}}\left[k_{p}^{2}-3 \frac{1-i k_{p}\left|\mathbf{r}-\mathbf{r}^{\prime}\right|}{\left|\mathbf{r}-\mathbf{r}^{\prime}\right|^{2}}\right] \\
& +\frac{e^{i k_{p}\left|\mathbf{r}-\mathbf{r}^{\prime}\right|}}{\omega^{2}\left|\mathbf{r}-\mathbf{r}^{\prime}\right|^{3}}\left(1-i k_{p}\left|\mathbf{r}-\mathbf{r}^{\prime}\right|\right) \delta_{k j} \\
& -\frac{e^{i k_{s}\left|\mathbf{r}-\mathbf{r}^{\prime}\right|}\left(x_{k}-x_{k}^{\prime}\right)\left(x_{j}-x_{j}^{\prime}\right)}{\omega^{2}\left|\mathbf{r}-\mathbf{r}^{\prime}\right|^{3}}\left[k_{s}^{2}-3 \frac{1-i k_{s}\left|\mathbf{r}-\mathbf{r}^{\prime}\right|}{\left|\mathbf{r}-\mathbf{r}^{\prime}\right|^{2}}\right] \\
& -\frac{e^{i k_{s} \mathbf{r}-\mathbf{r}^{\prime} \mid}}{\omega^{2} \mid \mathbf{r}-\mathbf{r}^{\prime} \beta^{3}}\left(1-i k_{s}\left|\mathbf{r}-\mathbf{r}^{\prime}\right|\right) \delta_{k j} .
\end{aligned}
$$

An exterior integral representation for the $k$ th component $u_{k}(\mathbf{r})$ of the scattered wave can be obtained by applying Formula (17) for a classical solution $\mathbf{u}(\mathbf{r})$ of (1) and the vector fields $\hat{\mathbf{x}}_{k} \cdot \tilde{\Gamma}\left(\mathbf{r}, \mathbf{r}^{\prime}\right), k=1,2,3$. The integration extends over a region $\Omega_{\varepsilon, R}$ which lies outside the scatterer, outside a small sphere of radius $\varepsilon$ centered at the point $r$ and inside a large sphere of radius $R$ that includes the scatterer and the observation point $\mathbf{r}$. In the limit as $\varepsilon \rightarrow 0+$ and $R \rightarrow+\infty$. Relations (15), (16) yield after some long calculations the following integral representations, which hold for every $\mathbf{r} \in V$ and $k=1,2,3$.

$$
\begin{aligned}
u_{k}(\mathbf{r})= & \frac{1}{4 \pi} \int_{s}\left[\mathbf{u}\left(\mathbf{r}^{\prime}\right) \cdot T_{\mathbf{r}^{\prime}}\left(\hat{\mathbf{x}}_{k} \cdot \tilde{\Gamma}\left(\mathbf{r}, \mathbf{r}^{\prime}\right)\right)\right. \\
& \left.-\left(\hat{\mathbf{x}}_{k} \cdot \tilde{\Gamma}\left(\mathbf{r}, \mathbf{r}^{\prime}\right)\right) \cdot T_{\mathbf{r}^{\prime}} \mathbf{u}\left(\mathbf{r}^{\prime}\right)\right] d S\left(\mathbf{r}^{\prime}\right) .
\end{aligned}
$$

Therefore the scattered field $\mathbf{u}(\mathbf{r})$ satisfies the representation

$$
\mathbf{u}(\mathbf{r})=\frac{1}{4 \pi} \int_{s}\left[\mathbf{u}\left(\mathbf{r}^{\prime}\right) \cdot T_{\mathbf{r}^{\prime}} \tilde{\Gamma}\left(\mathbf{r}, \mathbf{r}^{\prime}\right)-\tilde{\Gamma}\left(\mathbf{r}, \mathbf{r}^{\prime}\right) \cdot T_{\mathbf{r}^{\prime}} \mathbf{u}\left(\mathbf{r}^{\prime}\right)\right] d S\left(\mathbf{r}^{\prime}\right)
$$

for $\mathbf{r} \in V$. The representation (23) coincides with the one given by Pao and Varatharajulu [13] with the appropriate modifications. Since the plane wave $\boldsymbol{\Phi}$ is a solution of (1) which has no singularities in $\mathbf{R}^{3}$, Betti's third formula implies that

$$
\int_{s}\left[\boldsymbol{\Phi}\left(\mathbf{r}^{\prime}\right) \cdot T_{\mathbf{r}^{\prime}} \tilde{\Gamma}\left(\mathbf{r}, \mathbf{r}^{\prime}\right)-\tilde{\Gamma}\left(\mathbf{r}, \mathbf{r}^{\prime}\right) \cdot T_{\mathbf{r}^{\prime}} \boldsymbol{\Phi}\left(\mathbf{r}^{\prime}\right)\right] d S\left(\mathbf{r}^{\prime}\right)=\mathbf{0}
$$

Relations (23) and (24) provide the following integral representation for the total field $\Psi(\mathbf{r})$ in the exterior domain $V$,

$$
\boldsymbol{\Psi}(\mathbf{r})=\boldsymbol{\Phi}(\mathbf{r})+\frac{1}{4 \pi} \int_{s}\left[\boldsymbol{\Psi}\left(\mathbf{r}^{\prime}\right) \cdot T_{\mathbf{r}^{\prime}} \tilde{\Gamma}\left(\mathbf{r}, \mathbf{r}^{\prime}\right)-\tilde{\Gamma}\left(\mathbf{r}, \mathbf{r}^{\prime}\right) \cdot T_{\mathbf{r}^{\prime}} \boldsymbol{\Psi}\left(\mathbf{r}^{\prime}\right)\right] d S\left(\mathbf{r}^{\prime}\right),
$$


where $\Phi$ can be either the longitudinal plane wave $\Phi^{p}$ given by (8) or the transverse plane wave $\Phi^{s}$ given by (9). If the scatterer is rigid then $\Psi$ vanishes on $S$ and the corresponding integral representation assumes the form

$$
\boldsymbol{\Psi}(\mathbf{r})=\boldsymbol{\Phi}(\mathbf{r})-\frac{1}{4 \pi} \int_{s} \tilde{\Gamma}\left(\mathbf{r}, \mathbf{r}^{\prime}\right) \cdot T_{\mathbf{r}^{\prime}} \Psi\left(\mathbf{r}^{\prime}\right) d S\left(\mathbf{r}^{\prime}\right) .
$$

On the other hand a cavity is described by the vanishing of $T \Psi$ on $S$ and the corresponding integral representation becomes

$$
\boldsymbol{\Psi}(\mathbf{r})=\boldsymbol{\Phi}(\mathbf{r})+\frac{1}{4 \pi} \int_{s} \boldsymbol{\Psi}\left(\mathbf{r}^{\prime}\right) \cdot T_{\mathbf{r}^{\prime}} \tilde{\Gamma}\left(\mathbf{r}, \mathbf{r}^{\prime}\right) d S\left(\mathbf{r}^{\prime}\right) .
$$

Whenever $\mathbf{r}$ varies on $S$, relation (26) becomes an integral equation for the rigid scattering problem (1), (11), (15), (16), while relation (27) becomes an integral equation for the cavity scattering problem (1), (12), (15), (16). In any case the representations (26), (27) are very useful for the description of the far-field behaviour of the scattered wave.

4. The scattering amplitudes. Barratt and Collins [2], using the asymptotic relations proved by Kupradze [10], were able to show that as $r \rightarrow+\infty$ the longitudinal part $\mathbf{u}^{p}$ and the transverse part $\mathbf{u}^{s}$ of the scattered wave satisfy the relations:

$$
\mathbf{u}^{p}(\mathbf{r})=h_{r}(\boldsymbol{\vartheta}, \varphi) \hat{\mathbf{r}} \frac{e^{i k_{p} r}}{r}+O\left(\frac{1}{r^{2}}\right)
$$

and

$$
\mathbf{u}^{s}(\mathbf{r})=\left(h_{\vartheta}(\vartheta, \varphi) \hat{\boldsymbol{\vartheta}}+h_{\varphi}(\vartheta, \varphi) \hat{\varphi}\right) \frac{e^{i k_{s} r}}{r}+O\left(\frac{1}{r^{2}}\right)
$$

where $\hat{\mathbf{r}}, \hat{\boldsymbol{\vartheta}}, \hat{\boldsymbol{\varphi}}$ are the unit vectors of the spherical coordinates. The function $h_{r} \hat{\mathbf{r}}$ has the outgoing radial direction $\hat{\mathbf{r}}$ and denotes the scattering amplitude of the longitudinal wave $\mathbf{u}^{p}$. Similarly the function $h_{\vartheta} \hat{\boldsymbol{\vartheta}}+h_{\varphi} \hat{\boldsymbol{\varphi}}$ have a tangential direction and denotes the scattering amplitude of the transverse wave $\mathbf{u}^{s}$. The form of the radiation relations (28) and (29) reflects the fact that on the surface of a large sphere, far away from the scatterer, the scattered wave behaves like a spherically expanding wave whose longitudinal part propagates along the radius while its transverse part is polarized tangentially to the sphere. Integral representations for the scattering amplitudes over the surface of the scatterer will be derived in the sequel.

The fundamental dyadic $\tilde{\Gamma}$ is written as

$$
\tilde{\Gamma}\left(\mathbf{r}, \mathbf{r}^{\prime}\right)=\tilde{\Gamma}^{p}\left(\mathbf{r}, \mathbf{r}^{\prime}\right)+\tilde{\Gamma}^{s}\left(\mathbf{r}, \mathbf{r}^{\prime}\right)
$$

where

$$
\begin{aligned}
\tilde{\Gamma}^{p}\left(\mathbf{r}, \mathbf{r}^{\prime}\right)=\frac{e^{i k_{p} \mathbf{r}-\mathbf{r}^{\prime} \mid}}{\omega^{2}\left|\mathbf{r}-\mathbf{r}^{\prime}\right|^{\beta}}[ & k_{p}^{2}\left(\mathbf{r}-\mathbf{r}^{\prime}\right) \otimes\left(\mathbf{r}-\mathbf{r}^{\prime}\right) \\
& \left.+\left(1-i k_{p}\left|\mathbf{r}-\mathbf{r}^{\prime}\right|\right)\left(\tilde{\mathbf{I}}-3 \frac{\left(\mathbf{r}-\mathbf{r}^{\prime}\right) \otimes\left(\mathbf{r}-\mathbf{r}^{\prime}\right)}{\left|\mathbf{r}-\mathbf{r}^{\prime}\right|^{2}}\right)\right]
\end{aligned}
$$


describes the longitudinal part and

$$
\begin{aligned}
\tilde{\Gamma}^{s}\left(\mathbf{r}, \mathbf{r}^{\prime}\right)= & -\frac{e^{i k_{s} \mathbf{r}-\mathbf{r}^{\prime} \mid}}{\omega^{2}\left|\mathbf{r}-\mathbf{r}^{\prime}\right|^{3}}\left[k_{s}^{2}\left(\mathbf{r}-\mathbf{r}^{\prime}\right) \otimes\left(\mathbf{r}-\mathbf{r}^{\prime}\right)\right. \\
& \left.+\left(1-i k_{s}\left|\mathbf{r}-\mathbf{r}^{\prime}\right|\right)\left(\tilde{\mathbf{I}}-3 \frac{\left(\mathbf{r}-\mathbf{r}^{\prime}\right) \otimes\left(\mathbf{r}-\mathbf{r}^{\prime}\right)}{\left|\mathbf{r}-\mathbf{r}^{\prime}\right|^{2}}\right)\right] \\
& +\frac{k_{s}^{2} \frac{e^{i k_{s} \mathbf{r}-\mathbf{r}^{\prime} \mid}}{\omega^{2}} \tilde{\left|\mathbf{r}-\mathbf{r}^{\prime}\right|}}{}
\end{aligned}
$$

describes the transverse part. By means of the asymptotic relation

$$
\frac{\mathbf{r}-\mathbf{r}^{\prime}}{\left|\mathbf{r}-\mathbf{r}^{\prime}\right|}=\hat{\mathbf{r}}+O\left(\frac{1}{r}\right), \quad r \rightarrow \infty
$$

and

$$
\left|\mathbf{r}-\mathbf{r}^{\prime}\right|=r-\hat{\mathbf{r}} \cdot \mathbf{r}^{\prime}+O\left(\frac{1}{r}\right), \quad r \rightarrow \infty
$$

it is concluded that, as $r \rightarrow \infty$

$$
\tilde{\Gamma}^{p}\left(\mathbf{r}, \mathbf{r}^{\prime}\right)=\hat{\mathbf{r}} \otimes \hat{\mathbf{r}} \frac{e^{-i k_{p} \hat{\mathbf{r}} \cdot \mathbf{r}^{\prime}}}{c_{p}^{2}} \frac{e^{i k_{p} r}}{r}+O\left(\frac{1}{r^{2}}\right)
$$

and

$$
\tilde{\Gamma}^{s}\left(\mathbf{r}, \mathbf{r}^{\prime}\right)=(\tilde{\mathbf{I}}-\hat{\mathbf{r}} \otimes \hat{\mathbf{r}}) \frac{e^{-i k_{s} \hat{\mathbf{r}} \cdot \mathbf{r}^{\prime}}}{c_{s}^{2}} \frac{e^{i k_{s} r}}{r}+O\left(\frac{1}{r^{2}}\right)
$$

The dyadic $\hat{\mathbf{r}} \otimes \hat{\mathbf{r}}$ in (35) indicates the radial behaviour of the longitudinal part of $\tilde{\Gamma}$ far away from the scatterer. Similarly, the dyadic $\tilde{\mathbf{I}}-\hat{\mathbf{r}} \otimes \hat{\mathbf{r}}$ in (36) indicates the tangential behaviour of the transverse part of $\tilde{\Gamma}$ in the radiation region.

In order to apply the surface stress operator

$$
T_{\mathbf{r}^{\prime}}=2 \mu \hat{\mathbf{n}}^{\prime} \cdot \nabla_{\mathbf{r}^{\prime}}+\lambda \hat{\mathbf{n}}^{\prime} \nabla_{\mathbf{r}^{\prime}}+\mu \hat{\mathbf{n}}^{\prime} \times \nabla_{\mathbf{r}^{\prime}} \times
$$

on the fundamental dyadic $\tilde{\Gamma}\left(\mathbf{r}, \mathbf{r}^{\prime}\right)$ it is necessary to evaluate the asymptotic form of the gradient of $\tilde{\Gamma}$ with respect to the variable $\mathbf{r}^{\prime}$. This can be done by evaluating $\nabla_{\mathbf{r}^{\prime}} \tilde{\Gamma}\left(\mathbf{r}, \mathbf{r}^{\prime}\right)$ and then using the asymptotic relations (33) and (34) to obtain

$$
\begin{aligned}
\nabla_{\mathbf{r}^{\prime}} \tilde{\Gamma}\left(\mathbf{r}, \mathbf{r}^{\prime}\right)= & -\hat{\mathbf{r}} \otimes \hat{\mathbf{r}} \otimes \hat{\mathbf{r}} \frac{i k_{p}}{c_{p}^{2}} e^{-i k_{p} \hat{\mathbf{r}} \cdot \mathbf{r}^{\prime}} \frac{e^{i k_{p} r}}{r} \\
& -\hat{\mathbf{r}} \otimes(\tilde{\mathbf{I}}-\hat{\mathbf{r}} \otimes \hat{\mathbf{r}}) \frac{i k_{s}}{c_{s}^{2}} e^{-i k_{s} \hat{\mathbf{r}} \cdot \mathbf{r}^{\prime}} \frac{e^{i k_{s} r}}{r}+O\left(\frac{1}{r^{2}}\right)
\end{aligned}
$$


as $r \rightarrow \infty$. Taking the scalar and the vector invariants between the first two vectors of the triadic $\nabla_{\mathbf{r}^{\prime}} \tilde{\Gamma}$ it is concluded that as $r \rightarrow \infty$,

$$
\nabla_{\mathbf{r}^{\prime}} \cdot \tilde{\Gamma}\left(\mathbf{r}, \mathbf{r}^{\prime}\right)=-\hat{\mathbf{r}} \frac{i k_{p}}{c_{p}^{2}} e^{-i k_{p} \hat{\mathbf{r}} \cdot \mathbf{r}^{\prime}} \frac{e^{i k_{p} r}}{r}+O\left(\frac{1}{r^{2}}\right)
$$

and

$$
\nabla_{\mathbf{r}^{\prime}} \times \tilde{\Gamma}\left(\mathbf{r}, \mathbf{r}^{\prime}\right)=(\hat{\boldsymbol{\vartheta}} \otimes \hat{\boldsymbol{\varphi}}-\hat{\boldsymbol{\varphi}} \otimes \hat{\boldsymbol{\vartheta}}) \frac{i k_{s}}{c_{s}^{2}} e^{-i k_{s^{\prime}} \cdot \mathbf{r}^{\prime}} \frac{e^{i k_{s} r}}{r}+O\left(\frac{1}{r^{2}}\right)
$$

where we have used the following spherical representation of the identity dyadic

$$
\tilde{\mathbf{I}}=\hat{\mathbf{r}} \otimes \hat{\mathbf{r}}+\hat{\boldsymbol{\vartheta}} \otimes \hat{\boldsymbol{\vartheta}}+\hat{\boldsymbol{\varphi}} \otimes \hat{\boldsymbol{\varphi}}
$$

Substituting (38), (39) and (40) into the expression for $T_{\mathbf{r}^{\prime}} \tilde{\Gamma}\left(\mathbf{r}, \mathbf{r}^{\prime}\right)$ the following asymptotic form, as $r \rightarrow \infty$, is derived

$$
\begin{aligned}
T_{\mathbf{r}^{\prime}} \tilde{\Gamma}\left(\mathbf{r}, \mathbf{r}^{\prime}\right)= & -\hat{\mathbf{n}}^{\prime} \cdot(\lambda \tilde{\mathbf{I}}+2 \mu \hat{\mathbf{r}} \otimes \hat{\mathbf{r}}) \otimes \hat{\mathbf{r}} \frac{i k_{p}}{c_{p}^{2}} e^{-i k_{p} \hat{\mathbf{r}} \cdot \mathbf{r}^{\prime}} \frac{e^{i k_{p} r}}{r} \\
& -\mu\left[2\left(\hat{\mathbf{n}}^{\prime} \cdot \hat{\mathbf{r}}\right) \hat{\boldsymbol{\vartheta}}+\left(\hat{\mathbf{n}}^{\prime} \times \hat{\boldsymbol{\varphi}}\right)\right] \otimes \hat{\boldsymbol{\vartheta}} \frac{i k_{s}}{c_{s}^{2}} e^{-i k_{s} \hat{\mathbf{r}} \cdot \mathbf{r}^{\prime}} \frac{e^{i k_{s} r}}{r} \\
& -\mu\left[2\left(\hat{\mathbf{n}}^{\prime} \cdot \hat{\mathbf{r}}\right) \hat{\boldsymbol{\varphi}}-\left(\hat{\mathbf{n}}^{\prime} \times \hat{\boldsymbol{\vartheta}}\right)\right] \otimes \hat{\boldsymbol{\varphi}} \frac{i k_{s}}{c_{s}^{2}} e^{-i k_{s} \hat{\mathbf{r}} \cdot \mathbf{r}^{\prime}} \frac{e^{i k_{s} r}}{r} \\
& +O\left(\frac{1}{r^{2}}\right), \quad r \rightarrow \infty .
\end{aligned}
$$

Contracting from the left with $\Psi\left(\mathbf{r}^{\prime}\right)$ the asymptotic form of the dyadic $T_{\mathbf{r}^{\prime}} \tilde{\Gamma}\left(\mathbf{r}, \mathbf{r}^{\prime}\right)$ one ohtains the following expression as $r \rightarrow \infty$

$$
\begin{aligned}
& \Psi\left(\mathbf{r}^{\prime}\right) \cdot T_{\mathbf{r}^{\prime}} \tilde{\Gamma}\left(\mathbf{r}, \mathbf{r}^{\prime}\right) \\
& =k_{p}^{2}\left(\boldsymbol{\Psi}\left(\mathbf{r}^{\prime}\right) \otimes \hat{\mathbf{n}}^{\prime}\right):\left(\frac{\lambda}{\lambda+2 \mu} \tilde{\mathbf{I}}+\frac{2 \mu}{\lambda+2 \mu} \hat{\mathbf{r}} \otimes \hat{\mathbf{r}}\right) \hat{\mathbf{r}} e^{-i k_{p} \hat{\mathbf{r}} \cdot \mathbf{r}^{\prime}} h\left(k_{p} r\right)
\end{aligned}
$$

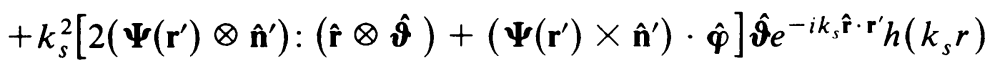

$$
\begin{aligned}
& +k_{s}^{2}\left[2\left(\boldsymbol{\Psi}\left(\mathbf{r}^{\prime}\right) \otimes \hat{\mathbf{n}}^{\prime}\right):(\hat{\mathbf{r}} \otimes \hat{\boldsymbol{\varphi}})-\left(\boldsymbol{\Psi}\left(\mathbf{r}^{\prime}\right) \times \hat{\mathbf{n}}^{\prime}\right) \cdot \hat{\boldsymbol{\vartheta}}\right] \hat{\boldsymbol{\varphi}} e^{-i k_{s} \hat{\mathbf{r}} \cdot \mathbf{r}^{\prime}} h\left(k_{s} r\right) \\
& +O\left(\frac{1}{r^{2}}\right)
\end{aligned}
$$

where $h(x)=e^{i x} / i x$ is the zeroth order spherical Hankel function of the first kind and the indicated double inner product is defined as

$$
(\mathbf{a} \otimes \mathbf{b}):(\mathbf{c} \otimes \mathbf{d})=(\mathbf{a} \cdot \mathbf{d})(\mathbf{b} \cdot \mathbf{c}) .
$$

Substituting (35), (36) and (43) into (25) it follows that as $r \rightarrow \infty$,

$$
\begin{aligned}
\mathbf{u}(\mathbf{r})= & g_{r}(\hat{\mathbf{r}}, \hat{\mathbf{k}}) \hat{\mathbf{r}} h\left(k_{p} r\right)+g_{\vartheta}(\hat{\mathbf{r}}, \hat{\mathbf{k}}) \hat{\boldsymbol{\vartheta}} h\left(k_{s} r\right) \\
& +g_{\varphi}(\hat{\mathbf{r}}, \hat{\mathbf{k}}) \hat{\boldsymbol{\varphi}} h\left(k_{s} r\right)+O\left(\frac{1}{r^{2}}\right)
\end{aligned}
$$


where the normalized spherical scattering amplitudes $g_{r}, g_{\vartheta}$ and $g_{\varphi}$ which describe the effect of the scatterer in the directions $\hat{\mathbf{r}}, \hat{\boldsymbol{\vartheta}}$ and $\hat{\boldsymbol{\varphi}}$ respectively, due to an incident plane wave propagating along $\hat{\mathbf{k}}$, are defined as follows

$$
\begin{gathered}
g_{r}(\hat{\mathbf{r}}, \hat{\mathbf{k}})=k_{p}^{2} \tilde{H}_{p}:\left(\frac{\lambda}{\lambda+2 \mu} \tilde{\mathbf{I}}+\frac{2 \mu}{\lambda+2 \mu} \hat{\mathbf{r}} \otimes \hat{\mathbf{r}}\right)-\frac{i k_{p}}{c_{p}^{2}}\left(\boldsymbol{l}_{p} \cdot \hat{\mathbf{r}}\right), \\
g_{\vartheta}(\hat{\mathbf{r}}, \hat{\mathbf{k}})=k_{s}^{2}\left[2 \tilde{H}_{s}: \hat{\mathbf{r}} \otimes \hat{\boldsymbol{\vartheta}}+\left(\mathbf{h}_{s} \cdot \hat{\boldsymbol{\varphi}}\right)\right]-\frac{i k_{s}}{c_{s}^{2}}\left(\boldsymbol{l}_{s} \cdot \hat{\boldsymbol{\vartheta}}\right), \\
g_{\varphi}(\hat{\mathbf{r}}, \hat{\mathbf{k}})=k_{s}^{2}\left[2 \tilde{H}_{s}: \hat{\mathbf{r}} \otimes \hat{\boldsymbol{\varphi}}-\left(\mathbf{h}_{s} \cdot \hat{\boldsymbol{\vartheta}}\right)\right]-\frac{i k_{s}}{c_{s}^{2}}\left(\boldsymbol{l}_{s} \cdot \hat{\boldsymbol{\varphi}}\right),
\end{gathered}
$$

and

$$
\begin{aligned}
\tilde{H}_{p} & =\frac{1}{4 \pi} \int_{s} \Psi\left(\mathbf{r}^{\prime}\right) \otimes \hat{\mathbf{n}}^{\prime} e^{-i k_{p} \hat{\mathbf{r}} \cdot \mathbf{r}^{\prime}} d S\left(\mathbf{r}^{\prime}\right), \\
\tilde{H}_{s} & =\frac{1}{4 \pi} \int_{s} \Psi\left(\mathbf{r}^{\prime}\right) \otimes \hat{\mathbf{n}}^{\prime} e^{-i k_{s} \hat{\mathbf{r}} \cdot \mathbf{r}^{\prime}} d S\left(\mathbf{r}^{\prime}\right), \\
\mathbf{h}_{s} & =\frac{1}{4 \pi} \int_{s} \Psi\left(\mathbf{r}^{\prime}\right) \otimes \hat{\mathbf{n}}^{\prime} e^{-i k_{s} \hat{\mathbf{r}} \cdot \mathbf{r}^{\prime}} d S\left(\mathbf{r}^{\prime}\right), \\
\boldsymbol{l}_{p} & =\frac{1}{4 \pi} \int_{s} T_{\mathbf{r}^{\prime}} \boldsymbol{\Psi}\left(\mathbf{r}^{\prime}\right) e^{-i k_{p} \hat{\mathbf{r}} \cdot \mathbf{r}^{\prime}} d S\left(\mathbf{r}^{\prime}\right), \\
\boldsymbol{l}_{s} & =\frac{1}{4 \pi} \int_{s} T_{\mathbf{r}^{\prime}} \boldsymbol{\Psi}\left(\mathbf{r}^{\prime}\right) e^{-i k_{s} \hat{\mathbf{r}} \cdot \mathbf{r}^{\prime}} d S\left(\mathbf{r}^{\prime}\right) .
\end{aligned}
$$

The vector $\mathbf{h}_{s}$ is the vector invariant of the dyadic $\tilde{H}_{s}$.

The advantage of the integral representations (46)-(53) for the normalized spherical scattering amplitudes $g_{r}, g_{\vartheta}$ and $g_{\varphi}$, over the corresponding representations of Barratt and Collins lies on the fact that (46)-(53) involve the values of $\Psi$ on $S$ and therefore the boundary conditions (12) or (13) are immediately applicable. On the other hand, due to the different wave numbers $k_{p}$ and $k_{s}$, the representations of Barratt and Collins cannot be combined in a straight forward manner in such a way that the total field $\Psi$ appears in the corresponding integrands.

The first term in the right-hand side of (45) corresponds to that part of the scattered wave which is due to the longitudinal wave and is directed along the radial direction $\hat{\mathbf{r}}$. Similarly the second and the third terms in the right hand side of (45) are due to the transverse wave and are polarized on a tangent (perpendicular to $\hat{\mathbf{r}}$ ) plane. This polarization vector $g_{\vartheta} \hat{\boldsymbol{v}}+g_{\varphi} \hat{\varphi}$ can also be written as follows

$$
\begin{aligned}
g_{\vartheta}(\hat{\mathbf{r}}, \hat{\mathbf{k}}) \hat{\boldsymbol{\vartheta}}+g_{\varphi}(\hat{\mathbf{r}}, \hat{\mathbf{k}}) \hat{\boldsymbol{\varphi}}= & g_{\iota}(\hat{\mathbf{r}}, \hat{\mathbf{k}}) \\
= & k_{s}^{2}\left[2 \tilde{H}_{s}: \hat{\mathbf{r}} \otimes(\tilde{\mathbf{I}}-\hat{\mathbf{r}} \otimes \hat{\mathbf{r}})+\mathbf{h}_{s} \times \hat{\mathbf{r}}\right] \\
& -\frac{i k_{s}}{c_{s}^{2}} \boldsymbol{l}_{s} \cdot(\tilde{\mathbf{I}}-\hat{\mathbf{r}} \otimes \hat{\mathbf{r}})
\end{aligned}
$$


where the representation

$$
\hat{\boldsymbol{\varphi}} \otimes \hat{\boldsymbol{\vartheta}}-\hat{\boldsymbol{\vartheta}} \otimes \hat{\boldsymbol{\varphi}}=\tilde{\mathbf{I}} \times \hat{\mathbf{r}}
$$

has been used.

The vector field $\mathbf{g}_{t}(\hat{\mathbf{r}}, \hat{\mathbf{k}})$ describes the tangential behaviour of the scattered wave in the region of radiation and can be used whenever the particular contributions to the directions $\hat{\boldsymbol{\vartheta}}$ and $\hat{\boldsymbol{\varphi}}$ are not important. It is the tangential normalized vector scattering amplitude. For a rigid scatterer $\Psi$ vanishes on $S$ and hence $\tilde{H}_{p}, \tilde{H}_{s}$ and $\mathbf{h}_{s}$ vanish too. In this case the three scattering amplitudes become

$$
\begin{aligned}
& g_{r}^{\text {rigid }}(\hat{\mathbf{r}}, \hat{\mathbf{k}})=-\frac{i k_{p}}{c_{p}^{2}}\left(\boldsymbol{l}_{p} \cdot \hat{\mathbf{r}}\right), \\
& g_{\vartheta}^{\text {rigid }}(\hat{\mathbf{r}}, \hat{\mathbf{k}})=-\frac{i k_{s}}{c_{s}^{2}}\left(\boldsymbol{l}_{s} \cdot \hat{\boldsymbol{\vartheta}}\right), \\
& g_{\varphi}^{\text {rigid }}(\hat{\mathbf{r}}, \hat{\mathbf{k}})=-\frac{i k_{s}}{c_{s}^{2}}\left(\boldsymbol{l}_{s} \cdot \hat{\boldsymbol{\varphi}}\right) .
\end{aligned}
$$

If the scattering region is a cavity, then $T \Psi$ vanishes on $S$ and so do the integrals $l_{p}$ and $\boldsymbol{l}_{s}$. The corresponding scattering amplitudes in this case are

$$
\begin{gathered}
g_{r}^{\text {cavity }}(\hat{\mathbf{r}}, \hat{\mathbf{k}})=k_{p}^{2} \tilde{H}_{p}:\left(\frac{\lambda}{\lambda+2 \mu} \tilde{\mathbf{I}}+\frac{2 \mu}{\lambda+2 \mu} \hat{\mathbf{r}} \otimes \hat{\mathbf{r}}\right), \\
g_{\vartheta}^{\text {cavity }}(\hat{\mathbf{r}}, \hat{\mathbf{k}})=k_{s}^{2}\left[2 \tilde{H}_{s}: \hat{\mathbf{r}} \otimes \hat{\boldsymbol{\vartheta}}+\left(\mathbf{h}_{s} \cdot \hat{\boldsymbol{\varphi}}\right)\right], \\
g_{\varphi}^{\text {cavity }}(\hat{\mathbf{r}}, \hat{\mathbf{k}})=k_{s}^{2}\left[2 \tilde{H}_{s}: \hat{\mathbf{r}} \otimes \hat{\boldsymbol{\varphi}}-\left(\mathbf{h}_{s} \cdot \hat{\boldsymbol{\vartheta}}\right)\right] .
\end{gathered}
$$

5. The scattering cross-section. The scattering cross-section is defined as the ratio of the time average rate (over a period) at which energy is scattered by the body, to the corresponding time average rate at which the energy of the incident wave crosses a unit area normal to the direction of propagation. The scattering cross-section is a measure of the disturbance caused by the scatterer to the incident wave. The rate at which the energy of the time harmonic displacement field $\mathbf{u}(\mathbf{r}) e^{-i \omega t}$ crosses a surface element with normal $\hat{\mathbf{n}}$ is given by

$$
-\operatorname{Re}\left\{T \mathbf{u}(\mathbf{r}) e^{-i \omega t}\right\} \frac{\partial}{\partial t} \operatorname{Re}\left\{\mathbf{u}(\mathbf{r}) e^{-i \omega t}\right\}
$$

where is the surface stress operator (13) evaluated in the normal direction $\hat{\mathbf{n}}$.

Calculating the time average of (62) over a period $2 \pi / \omega$ for the incident waves $\boldsymbol{\Phi}^{p}(\mathbf{r}) e^{-i \omega t}$ and $\boldsymbol{\Phi}^{s}(\mathbf{r}) e^{-i \omega t}$, where $\boldsymbol{\Phi}^{p}, \boldsymbol{\Phi}^{s}$ are given by (8) and (9) respectively, it is obtained that

$$
\begin{aligned}
e^{p} & =\frac{\omega}{2} \operatorname{Im}\left[\left(T \boldsymbol{\Phi}^{p}\right) \cdot\left(\boldsymbol{\Phi}^{p}\right)^{*}\right]=\frac{\omega^{3}}{2 k_{p}}, \\
e^{s} & =\frac{\omega}{2} \operatorname{Im}\left[\left(T \boldsymbol{\Phi}^{s}\right) \cdot\left(\boldsymbol{\Phi}^{s}\right)^{*}\right]=\frac{\omega^{3}}{2 k_{s}},
\end{aligned}
$$


where the star indicates complex conjugation and $e^{p}, e^{s}$ are the time average rate at which the incident $P$ and $S$ plane waves respectively cross a unit area normal to the direction of propagation $\hat{\mathbf{k}}$.

On the other hand, the time average rate at which energy is scattered by the body is the time average rate at which the energy of the scattered field is transmitted across a large sphere $S_{r}, r \gg 1$, enclosing the scatterer. As before, if $\mathbf{u}(\mathbf{r}) e^{-i \omega t}$ is the displacement field for the scattered wave the stress vector on the surface of the sphere $S_{r}$, at the point $\mathbf{r}$ is given by $T \mathbf{u}(\mathbf{r}) e^{-i \omega t}$, where $T$ is the surface stress operator with $\hat{\mathbf{n}}=\hat{\mathbf{r}}$. The rate at which the energy of the scattered field is transmitted across $S_{r}$ is given by the integral

$$
-\int_{S_{r}} \operatorname{Re}\left\{T \mathbf{u}(\mathbf{r}) e^{-i \omega t}\right\} \frac{\partial}{\partial t} \operatorname{Re}\left\{\mathbf{u}(\mathbf{r}) e^{-i \omega t}\right\} d S .
$$

Evaluating the corresponding time average over a period it is concluded that

$$
e=\frac{\omega}{2} \operatorname{Im} \int_{S_{r}}(T \mathbf{u}(\mathbf{r})) \cdot \mathbf{u}^{*}(\mathbf{r}) d S .
$$

On the surface $S_{r}$ the traction has the asymptotic form, as $r \rightarrow \infty$,

$$
\begin{aligned}
T \mathbf{u}(\mathbf{r}) & =2 \mu \hat{\mathbf{r}} \cdot \nabla \mathbf{u}(\mathbf{r})+\lambda \hat{\mathbf{r}} \nabla \cdot \mathbf{u}(\mathbf{r})+\mu \hat{\mathbf{r}} \times(\nabla \times \mathbf{u}(\mathbf{r})) \\
& =(\lambda+2 \mu) g_{r} \hat{\mathbf{r}} \frac{e^{i k_{p} r}}{r}+\mu\left(g_{\vartheta} \hat{\boldsymbol{\vartheta}}+g_{\varphi} \hat{\boldsymbol{\varphi}}\right) \frac{e^{i k, r}}{r}+O\left(\frac{1}{r^{2}}\right)
\end{aligned}
$$

which, in view of (45), gives

$$
(T \mathbf{u}(\mathbf{r})) \cdot\left(\mathbf{u}^{*}(\mathbf{r})\right)=i \frac{\lambda+2 \mu}{k_{p} r^{2}}\left|g_{r}\right|^{2}+i \frac{\mu}{k_{s} r^{2}}\left(\left|g_{\vartheta}\right|^{2}+\left|g_{\varphi}\right|^{2}\right) .
$$

Substituting (68) into (66) it is concluded that

$$
e(\hat{\mathbf{k}})=\frac{1}{2} \int_{\mid \hat{\mathbf{r}}=1}\left[c_{p}^{3}\left|g_{r}(\hat{\mathbf{r}}, \hat{\mathbf{k}})\right|^{2}+c_{s}^{3}\left(\left|g_{\vartheta}(\hat{\mathbf{r}}, \hat{\mathbf{k}})\right|^{2}+\left|g_{\varphi}(\hat{\mathbf{r}}, \hat{\mathbf{k}})\right|^{2}\right)\right] d \Omega(\hat{\mathbf{r}}),
$$

where the integration is taken over the unit sphere. From (63), (64) and (69) the scattering cross-sections $\sigma^{p}$ and $\sigma^{s}$, corresponding to an incident $P$ and $S$ wave respectively are expressed as follow

$$
\begin{aligned}
& \boldsymbol{\sigma}^{p}=\frac{e(\hat{\mathbf{k}})}{e^{p}}=k_{p} \int_{\mid \hat{\boldsymbol{r}}=1}\left[k_{p}^{-3}\left|g_{r}\right|^{2}+k_{s}^{-3}\left(\left|g_{\vartheta}\right|^{2}+\left|g_{\varphi}\right|^{2}\right)\right] d \Omega(\hat{\mathbf{r}}), \\
& \boldsymbol{\sigma}^{s}=\frac{e(\hat{\mathbf{k}})}{e^{s}}=k_{s} \int_{\mid \hat{\mid} \boldsymbol{|}=1}\left[k_{p}^{-3}\left|g_{r}\right|^{2}+k_{s}^{-3}\left(\left|g_{\vartheta}\right|^{2}+\left|g_{\varphi}\right|^{2}\right)\right] d \Omega(\hat{\mathbf{r}}) .
\end{aligned}
$$

Obviously, the integrals that appear in the right-hand side of (70) and (71) are not the same since the amplitudes $g_{r}, g_{\vartheta}$ and $g_{\varphi}$, which correspond to longitudinal and transverse incidence, differ. 
Dimensionalwise, since the normalized spherical scattering amplitudes $g_{r}, g_{\vartheta}, g_{\varphi}$ are dimensionless, it is concluded that the scattering cross-section $\sigma^{p}$ or $\sigma^{s}$ have the dimensions of area, i.e.

$$
[\sigma]=\left[L^{2}, M^{0}, T^{0}\right]
$$

and this fact justifies the characterization of $\sigma$ as cross-section.

Using Jones' Lemma for the asymptotic evaluation of double integrals, Barratt and Collins [2] have expressed the scattering cross-sections by means of the values of the spherical scattering amplitudes in the forward direction. Their results translated to our terminology read as follow

$$
\boldsymbol{\sigma}^{p}=-\frac{4 \pi}{k_{p}^{2}} \operatorname{Re} g_{r}(\hat{\mathbf{k}}, \hat{\mathbf{k}})
$$

and

$$
\sigma^{s}=-\frac{4 \pi}{k_{s}^{2}} \operatorname{Re}\left\{g_{\vartheta}(\hat{\mathbf{k}}, \hat{\mathbf{k}})(\hat{\mathbf{b}} \cdot \hat{\boldsymbol{\vartheta}})+g_{\varphi}(\hat{\mathbf{k}}, \hat{\mathbf{k}})(\hat{\mathbf{b}} \cdot \hat{\boldsymbol{\varphi}})\right\}
$$

where $\hat{\mathbf{b}}$ is the polarization vector of the incident $S$-wave.

6. The low-frequency theory. It is well known that the solutions of the time-independent Navier Equation (1), considered as functions of the wave number $k_{s}$ or $k_{p}$, are analytic in a neighborhood of zero. As a consequence, the displacement field can be expanded in a convergent power series of the wave number $k_{s}$ or $k_{p}$. For convenience, as well as comparison purposes, the following terminology is introduced

$$
\left.\begin{array}{r}
k=k_{s} \\
\tau^{2}=\frac{\mu}{\lambda+2 \mu}=\frac{k_{p}^{2}}{k_{s}^{2}}
\end{array}\right\} .
$$

Then the total field $\Psi$ satisfies the equation

$$
\tau^{2} \Delta \Psi(\mathbf{r})+\left(1-\tau^{2}\right) \nabla(\nabla \cdot \Psi(\mathbf{r}))+\tau^{2} k^{2} \Psi(\mathbf{r})=\mathbf{0} .
$$

Inserting the expansion

$$
\boldsymbol{\Psi}(\mathbf{r})=\sum_{n=0}^{\infty} \frac{\left(i k_{p}\right)^{n}}{n !} \boldsymbol{\Phi}_{n}(\mathbf{r})=\sum_{n=0}^{\infty} \frac{(i \tau k)^{n}}{n !} \boldsymbol{\Phi}_{n}(\mathbf{r})
$$

into (76) and equating equal powers of $k$ the following sequence of partial differential equations is obtained

$$
\tau^{2} \Delta \boldsymbol{\Phi}_{n}(\mathbf{r})+\left(1-\tau^{2}\right) \nabla \nabla \cdot \boldsymbol{\Phi}_{n}(\mathbf{r})=n(n-1) \boldsymbol{\Phi}_{n-2}(\mathbf{r})
$$

for $n=0,1,2, \ldots$ 
The expansion (77) separates the spatial from the wave number dependence of the solution. In fact equation (78) describes the spatial dependence of $\Psi$ that corresponds to the $n$th power of $k$. For $n=0$ and $n=1$ equation (78) coincides with the static Navier equation in the absence of body forces, while for $n \geqslant 2$ it corresponds to the static Navier equation with a body force proportional to the $(n-2)$ th order coefficient which is known from previous steps. The boundary conditions (11) and (12) are transformed into the boundary conditions

$$
\boldsymbol{\Phi}_{n}(\mathbf{r})=\mathbf{0}, \quad \mathbf{r} \in S, n=0,1,2, \ldots
$$

and

$$
T \boldsymbol{\Phi}_{n}(\mathbf{r})=\mathbf{0}, \quad \mathbf{r} \in S, n=0,1,2, \ldots
$$

for the rigid scatterer and the cavity respectively. The incident waves can also be expanded into convergent power series of $k$ as follows

$$
\boldsymbol{\Phi}^{p}(\mathbf{r})=\hat{\mathbf{k}} \sum_{n=0}^{\infty} \frac{(i \tau k)^{n}}{n !}(\hat{\mathbf{k}} \cdot \mathbf{r})^{n}
$$

and

$$
\boldsymbol{\Phi}^{s}(\mathbf{r})=\hat{\mathbf{b}} \sum_{n=0}^{\infty} \frac{(i \tau k)^{n}}{\tau^{n} n !}(\hat{\mathbf{k}} \cdot \mathbf{r})^{n}
$$

In order to find the low-frequency expansion of the fundamental dyadic $\tilde{\Gamma}\left(\mathbf{r}, \mathbf{r}^{\prime}\right)$ it is observed that

$$
\begin{aligned}
\tilde{\Gamma}\left(\mathbf{r}, \mathbf{r}^{\prime}\right) & =\frac{1}{\mu} \frac{e^{i k\left|\mathbf{r}-\mathbf{r}^{\prime}\right|}}{\left|\mathbf{r}-\mathbf{r}^{\prime}\right|} \tilde{\mathbf{I}}-\frac{1}{\mu k^{2}} \nabla_{\mathbf{r}} \nabla_{\mathbf{r}}\left(\frac{e^{i \tau k\left|\mathbf{r}-\mathbf{r}^{\prime}\right|}}{\left|\mathbf{r}-\mathbf{r}^{\prime}\right|}-\frac{e^{i k\left|\mathbf{r}-\mathbf{r}^{\prime}\right|}}{\left|\mathbf{r}-\mathbf{r}^{\prime}\right|}\right) \\
& =\frac{1}{\mu} \tilde{\mathbf{I}} \sum_{n=0}^{\infty} \frac{(i k)^{n}}{n !}\left|\mathbf{r}-\mathbf{r}^{\prime}\right|^{n-1}+\frac{1}{\mu} \sum_{n=0}^{\infty} \frac{(i k)^{n-2}}{n !}\left(\tau^{n}-1\right) \nabla_{\mathbf{r}} \nabla_{\mathbf{r}}\left|\mathbf{r}-\mathbf{r}^{\prime}\right|^{n-1} .
\end{aligned}
$$

In the last sum, the term that corresponds to $n=0$ is zero since $\tau^{0}-1=0$. The term $n=1$ is also zero since $\nabla_{\mathbf{r}} \nabla_{\mathbf{r}}\left|\mathbf{r}-\mathbf{r}^{\prime}\right|^{1-1}=0$. Furthermore, for $n \geqslant 2$,

$$
\nabla_{\mathbf{r}} \nabla_{\mathbf{r}}\left|r-r^{\prime}\right|^{n-1}=(n-1)\left[\tilde{\mathbf{I}}+(n-3) \frac{\left(\mathbf{r}-\mathbf{r}^{\prime}\right) \otimes\left(\mathbf{r}-\mathbf{r}^{\prime}\right)}{\left|\mathbf{r}-\mathbf{r}^{\prime}\right|^{2}}\right]\left|\mathbf{r}-\mathbf{r}^{\prime}\right|^{n-3}
$$

Finally the fundamental dyadic has the expansion

$$
\tilde{\Gamma}\left(\mathbf{r}, \mathbf{r}^{\prime}\right)=\frac{1}{\mu} \sum_{n=0}^{\infty} \frac{(i \tau k)^{n}}{n !} \tilde{\gamma}_{n}\left(\mathbf{r}, \mathbf{r}^{\prime}\right)
$$


where

$$
\tilde{\gamma}_{n}\left(\mathbf{r}, \mathbf{r}^{\prime}\right)=\frac{\left|\mathbf{r}-\mathbf{r}^{\prime}\right|^{n-1}}{\tau^{n}}\left[\left(i+\frac{\tau^{n+2}-1}{n+2}\right) \tilde{\mathbf{I}}+(n-1) \frac{\tau^{n+2}-1}{n+2} \frac{\left(\mathbf{r}-\mathbf{r}^{\prime}\right) \otimes\left(\mathbf{r}-\mathbf{r}^{\prime}\right)}{\left|r-r^{\prime}\right|^{2}}\right]
$$

for $n=0,1,2, \ldots$ From $(77)$ and (86) it is derived that

$$
T \Psi(\mathbf{r})=\sum_{n=0}^{\infty} \frac{(i \tau k)^{n}}{n !} T \boldsymbol{\Phi}_{n}(\mathbf{r})
$$

and

$$
T_{\mathbf{r}^{\prime}} \tilde{\Gamma}\left(\mathbf{r}, \mathbf{r}^{\prime}\right)=\frac{1}{\mu} \sum_{n=0}^{\infty} \frac{(i \tau k)^{n}}{n !} T_{\mathbf{r}^{\prime}} \tilde{\gamma}_{n}\left(\mathbf{r}, \mathbf{r}^{\prime}\right)
$$

where

$$
\begin{aligned}
T_{\mathbf{r}^{\prime}} \tilde{\gamma}_{n}\left(\mathbf{r}, \mathbf{r}^{\prime}\right)= & -\frac{(n-1)}{\tau^{n}(n+2)}\left|\mathbf{r}-\mathbf{r}^{\prime}\right|^{n-2} \\
& \cdot\left\{\mu\left(2 \tau^{n+2}+n\right)\left[\frac{\hat{\mathbf{n}}^{\prime} \cdot\left(\mathbf{r}-\mathbf{r}^{\prime}\right)}{\left|\mathbf{r}-\mathbf{r}^{\prime}\right|} \tilde{\mathbf{I}}+\frac{\left(\mathbf{r}-\mathbf{r}^{\prime}\right) \otimes \hat{\mathbf{n}}^{\prime}}{\left|\mathbf{r}-\mathbf{r}^{\prime}\right|}\right]\right. \\
& +2 \mu(n-3)\left(\tau^{n+2}-1\right) \frac{\hat{\mathbf{n}}^{\prime} \cdot\left(\mathbf{r}-\mathbf{r}^{\prime}\right)}{\left|\mathbf{r}-\mathbf{r}^{\prime}\right|} \frac{\left(\mathbf{r}-\mathbf{r}^{\prime}\right) \otimes\left(\mathbf{r}-\mathbf{r}^{\prime}\right)}{\left|\mathbf{r}-\mathbf{r}^{\prime}\right|^{2}} \\
& \left.+\left[\lambda(n+2) \tau^{n+2}+2 \mu\left(\tau^{n+2}-1\right)\right] \frac{\hat{\mathbf{n}}^{\prime} \otimes\left(\mathbf{r}-\mathbf{r}^{\prime}\right)}{\left|\mathbf{r}-\mathbf{r}^{\prime}\right|}\right\} .
\end{aligned}
$$

The expression in brackets in (89) involves only the unit vector $\left(\mathbf{r}-\mathbf{r}^{\prime}\right) /\left|\mathbf{r}-\mathbf{r}^{\prime}\right|$ and therefore is bounded in $r$. Hence, as $r \rightarrow+\infty$ the order of $T_{\mathbf{r}}, \tilde{\gamma}_{n}\left(\mathbf{r}, \mathbf{r}^{\prime}\right)$ coincides with the order of $r^{n-2}$. In particular $T_{\mathbf{r}}, \tilde{\gamma}_{0}$ and $T_{\mathbf{r}}, \tilde{\gamma}_{1}$ vanish as $r \rightarrow+\infty$. Substituting (77), (81), (82), (85), (87) and (88) into (25) and equating equal powers of $k$, the following integral relations among the coefficients $\boldsymbol{\Phi}_{0}, \boldsymbol{\Phi}_{1}, \ldots, \boldsymbol{\Phi}_{n}$ are obtained

$$
\begin{aligned}
\boldsymbol{\Phi}_{n}(\mathbf{r})=\boldsymbol{\alpha}_{n}(\hat{\mathbf{k}} \cdot \mathbf{r})^{n}+\frac{1}{4 \pi \mu} \sum_{\rho=0}^{n}\left(\begin{array}{l}
n \\
\rho
\end{array}\right) \int_{s} & {\left[\Phi_{\rho}\left(\mathbf{r}^{\prime}\right) \cdot T_{\mathbf{r}^{\prime}} \tilde{\gamma}_{n-\rho}\left(\mathbf{r}, \mathbf{r}^{\prime}\right)\right.} \\
& \left.-\tilde{\gamma}_{\rho}\left(\mathbf{r}, \mathbf{r}^{\prime}\right) \cdot T_{\mathbf{r}^{\prime}} \boldsymbol{\Phi}_{n-\rho}\left(\mathbf{r}^{\prime}\right)\right] d S\left(\mathbf{r}^{\prime}\right)
\end{aligned}
$$

where

$$
\boldsymbol{\alpha}_{n}= \begin{cases}\hat{\mathbf{k}}, & \text { when } \boldsymbol{\Phi}=\boldsymbol{\Phi}^{p} \\ \frac{\hat{\mathbf{b}}}{\tau^{n}}, & \text { when } \boldsymbol{\Phi}=\boldsymbol{\Phi}^{s}\end{cases}
$$


and $\tilde{\gamma}_{n}\left(\mathbf{r}, \mathbf{r}^{\prime}\right), T_{\mathbf{r}^{\prime}} \tilde{\gamma}_{n}\left(\mathbf{r}, \mathbf{r}^{\prime}\right)$ are given by (89), (92) respectively. Since

$$
\tilde{\gamma}_{0}\left(\mathbf{r}, \mathbf{r}^{\prime}\right)=O\left(\frac{1}{r}\right), \quad r \rightarrow+\infty
$$

the integral relation (90) assumes the following asymptotic integral representation for the $n$th coefficient

$$
\begin{aligned}
\boldsymbol{\Phi}_{n}(\mathbf{r})= & \boldsymbol{\alpha}_{n}(\hat{\mathbf{k}} \cdot \mathbf{r})^{n} \\
& +\frac{1}{4 \pi \mu} \sum_{\rho=0}^{n-1}\left(\begin{array}{l}
n \\
\rho
\end{array}\right) \int\left[\Phi_{\rho}\left(\mathbf{r}^{\prime}\right) \cdot T_{\mathbf{r}^{\prime}} \tilde{\gamma}_{n-\rho}\left(\mathbf{r}, \mathbf{r}^{\prime}\right)-\tilde{\gamma}_{n-\rho}\left(\mathbf{r}, \mathbf{r}^{\prime}\right) \cdot T_{\mathbf{r}^{\prime}} \boldsymbol{\Phi}_{\rho}\left(\mathbf{r}^{\prime}\right)\right] d S\left(\mathbf{r}^{\prime}\right) \\
& +O\left(\frac{1}{r}\right), \quad r \rightarrow+\infty
\end{aligned}
$$

By straightforward calculations it can be shown that the nonvanishing part of the asymptotic expression (93) satisfies equation (78). Consequently a particular solution of the nonhomogeneous equation (78) is provided by

$$
\begin{aligned}
\mathbf{P}_{n}(\mathbf{r})= & \boldsymbol{\alpha}_{n}(\hat{\mathbf{k}} \cdot \mathbf{r})^{n} \\
& +\frac{1}{4 \pi \mu} \sum_{\rho=0}^{n-1}\left(\begin{array}{l}
n \\
\rho
\end{array}\right) \int_{s}\left[\boldsymbol{\Phi}_{\rho}\left(\mathbf{r}^{\prime}\right) \cdot T_{\mathbf{r}^{\prime}} \tilde{\gamma}_{n-\rho}\left(\mathbf{r}, \mathbf{r}^{\prime}\right)-\tilde{\gamma}_{n-\rho}\left(\mathbf{r}, \mathbf{r}^{\prime}\right) \cdot T_{\mathbf{r}^{\prime}} \mathbf{\Phi}_{\rho}\left(\mathbf{r}^{\prime}\right)\right] d S\left(\mathbf{r}^{\prime}\right)
\end{aligned}
$$

Since $\tilde{\gamma}_{1}\left(\mathbf{r}, \mathbf{r}^{\prime}\right)$ is a constant dyadic the $\rho=n-1$ term in (94) can be omitted without effecting the particular solution of (78). Nevertheless keeping the term $\rho=n-1$ in the sum $\mathbf{P}_{n}(\mathbf{r})$ provides both a particular solution of (78) and the asymptotic behaviour of $\boldsymbol{\Phi}_{n}(\mathbf{r})$ as $r \rightarrow+\infty$. In particular for the case of a cavity the $\rho=n-1$ term can always be omitted since $T_{\mathbf{r}} \tilde{\gamma}_{1}\left(\mathbf{r}, \mathbf{r}^{\prime}\right)$ is of the order of $1 / r$ as $r \rightarrow+\infty$. The solution of (78) is now written as

$$
\boldsymbol{\Phi}_{n}(\mathbf{r})=\mathbf{P}_{n}(\mathbf{r})+\mathbf{U}_{n}(\mathbf{r})
$$

where the function $\mathbf{P}_{n}(\mathbf{r})$, given by (94) satisfies (78) and describes the nonvanishing behaviour of $\boldsymbol{\Phi}_{n}(\mathbf{r})$, as $r \rightarrow+\infty$. The function $\mathbf{U}_{n}(\mathbf{r})$ satisfies the homogeneous equation

$$
\tau^{2} \Delta \mathbf{U}_{n}(\mathbf{r})+\left(1-\tau^{2}\right) \nabla \nabla \cdot \mathbf{U}_{n}(\mathbf{r})=\mathbf{0}
$$

the boundary condition

$$
L \mathbf{U}_{n}(\mathbf{r})=-L \mathbf{P}_{n}(\mathbf{r}), \quad \mathbf{r} \in S,
$$

where $L$ is the identity operator when $S$ is rigid and the surface stress operator $T$ when the scatterer is a cavity and for $r \rightarrow+\infty$

$$
\mathbf{U}(\mathbf{r})=O\left(\frac{1}{r}\right)
$$


Introducing the vector and the scalar Papkovich potentials $A^{n}$ and $B^{n}$ through the representation

$$
\mathbf{U}_{n}(\mathbf{r})=\mathbf{A}^{n}+\frac{1}{2}\left(\tau^{2}-1\right) \nabla\left(\mathbf{r} \cdot \mathbf{A}^{n}+B^{n}\right), \quad n=0,1,2, \ldots
$$

and substituting into (96) it is concluded that

$$
\begin{aligned}
& \Delta \mathbf{A}^{n}=\mathbf{0}, \quad n=0,1,2, \ldots, \\
& \Delta B^{n}=0, \quad n=0,1,2, \ldots
\end{aligned}
$$

It is well known that the potentials $\mathbf{A}^{n}$ and $B^{n}$ for the homogeneous equation (96) are not independent. As a matter of fact the vector potential $\mathbf{A}^{n}$ alone suffices to solve Equation (96). Nevertheless, in applications [7, 8], it is crucial to introduce both potentials $\mathbf{A}^{n}$ and $B^{n}$ and to use their dependence in order to be able to find closed form solutions for the corresponding potential problems. Recapitulating, the steps one has to follow in order to evaluate the $n$th coefficient $\boldsymbol{\Phi}_{n}$ for the low-frequency expansion of the displacement field are: (a) evaluate the integrals in (94) to find $\mathbf{P}_{n}(\mathbf{r})$ and (b) find harmonic functions $\mathbf{A}^{n}$ and $B^{n}$ such that

$$
L\left[\mathbf{A}^{n}(\mathbf{r})+\frac{1}{2}\left(\tau^{2}-1\right) \nabla\left(\mathbf{r} \cdot \mathbf{A}^{n}(\mathbf{r})+B^{n}(\mathbf{r})\right)\right]=-L \mathbf{P}_{n}(\mathbf{r}), \quad \mathbf{r} \in S
$$

and

$$
\mathbf{A}^{n}(\mathbf{r})+\frac{1}{2}\left(\tau^{2}-1\right) \nabla\left(\mathbf{r} \cdot \mathbf{A}^{n}(\mathbf{r})+B^{n}(\mathbf{r})\right)=O\left(\frac{1}{r}\right), \quad r \rightarrow+\infty .
$$

Then the $n$th coefficient is given by

$$
\boldsymbol{\Phi}_{n}(\mathbf{r})=\mathbf{P}_{n}(\mathbf{r})+\mathbf{A}^{n}(\mathbf{r})+\frac{1}{2}\left(\tau^{2}-1\right) \nabla\left(\mathbf{r} \cdot \mathbf{A}^{n}(\mathbf{r})+B^{n}(\mathbf{r})\right) .
$$

In particular, for the first two coefficients

$$
\begin{aligned}
\mathbf{P}_{0}(\mathbf{r})= & \boldsymbol{\alpha}_{0} \\
\mathbf{P}_{1}(\mathbf{r})= & \boldsymbol{\alpha}_{1}(\hat{\mathbf{k}} \cdot \mathbf{r}) \\
& +\frac{1}{4 \pi \mu} \int\left[\Phi_{0}\left(\mathbf{r}^{\prime}\right) \cdot T_{\mathbf{r}^{\prime}} \tilde{\gamma}_{1}\left(\mathbf{r}, \mathbf{r}^{\prime}\right)-\tilde{\gamma}_{1}\left(\mathbf{r}, \mathbf{r}^{\prime}\right) \cdot T_{\mathbf{r}^{\prime}} \boldsymbol{\Phi}_{0}\left(\mathbf{r}^{\prime}\right)\right] d S\left(\mathbf{r}^{\prime}\right) .
\end{aligned}
$$

Since

$$
\tilde{\gamma}_{1}\left(\mathbf{r}, \mathbf{r}^{\prime}\right)=\frac{\tau^{3}+2}{3 \tau} \tilde{\mathbf{I}}
$$

the functions $\mathbf{P}_{0}, \mathbf{P}_{1}$ for the rigid scatterer become

$$
\begin{aligned}
& \mathbf{P}_{0}^{\text {rigid }}(\mathbf{r})=\boldsymbol{\alpha}_{0}, \\
& \mathbf{P}_{1}^{\text {rigid }}(\mathbf{r})=\boldsymbol{\alpha}_{1}(\hat{\mathbf{k}} \cdot \mathbf{r})-\frac{\tau^{3}+2}{12 \pi \mu \tau} \int_{s} T_{\mathbf{r}^{\prime}} \boldsymbol{\Phi}_{0}\left(r^{\prime}\right) d S\left(\mathbf{r}^{\prime}\right)
\end{aligned}
$$


and for the cavity

$$
\begin{aligned}
& \mathbf{P}_{0}^{\text {cavity }}(\mathbf{r})=\boldsymbol{\alpha}_{0}, \\
& \mathbf{P}_{1}^{\text {cavity }}(\mathbf{r})=\boldsymbol{\alpha}_{1}(\hat{\mathbf{k}} \cdot \mathbf{r}),
\end{aligned}
$$

where $\boldsymbol{\alpha}_{0}, \boldsymbol{\alpha}_{1}$ are given by (91).

In particular, the solution $\boldsymbol{\Phi}_{0}$ for the cavity is always equal to $\mathbf{a}_{0}$, as it is given by (91). Indeed the constant function $\boldsymbol{\Phi}_{0}=\mathbf{a}_{0}$ satisfies, for $n=0$, Eq. (78), and the conditions (80) and (93). The uniqueness theorem implies that there is no other solution.

7. The far-field behaviour at low-frequency. In order to derive the low-frequency expansions for the scattering amplitudes $g_{r}, g_{\vartheta}$ and $g_{\varphi}$, the expansions (77), (87) and

$$
\begin{aligned}
e^{-i k_{p} \hat{\mathbf{r}} \cdot \mathbf{r}^{\prime}} & =\sum_{n=0}^{\infty}(-1)^{n} \frac{(i \tau k)^{n}}{n !}\left(\hat{\mathbf{r}} \cdot \mathbf{r}^{\prime}\right)^{n}, \\
e^{-i k_{s} \hat{\mathbf{r}} \cdot \mathbf{r}^{\prime}} & =\sum_{n=0}^{\infty}(-1)^{n} \frac{(i \tau k)^{n}}{\tau^{n} n !}\left(\hat{\mathbf{r}} \cdot \mathbf{r}^{\prime}\right)^{n},
\end{aligned}
$$

are substituted into (49)-(53) to obtain

$$
\begin{aligned}
\tilde{H}_{p} & =\frac{1}{4 \pi} \sum_{n=0}^{\infty} \frac{(i \tau k)^{n}}{n !} \sum_{\rho}^{n}\left(\begin{array}{l}
n \\
\rho
\end{array}\right)(-1)^{\rho} \int_{s} \boldsymbol{\Phi}_{n-\rho}\left(\mathbf{r}^{\prime}\right) \otimes \hat{\mathbf{n}}^{\prime}\left(\hat{\mathbf{r}} \cdot \mathbf{r}^{\prime}\right)^{\rho} d S\left(\mathbf{r}^{\prime}\right), \\
\tilde{H}_{s} & =\frac{1}{4 \pi} \sum_{n=0}^{\infty} \frac{(i \tau k)^{n}}{n !} \sum_{\rho=0}^{n}\left(\begin{array}{l}
n \\
\rho
\end{array}\right)\left(-\frac{1}{\tau}\right)^{\rho} \int_{s} \boldsymbol{\Phi}_{n-\rho}\left(\mathbf{r}^{\prime}\right) \otimes \hat{\mathbf{n}}^{\prime}\left(\hat{\mathbf{r}} \cdot \mathbf{r}^{\prime}\right)^{\rho} d S\left(\mathbf{r}^{\prime}\right), \\
\mathbf{h}_{s} & =\frac{1}{4 \pi} \sum_{n=0}^{\infty} \frac{(i \tau k)^{n}}{n !} \sum_{\rho=0}^{n}\left(\begin{array}{l}
n \\
\rho
\end{array}\right)\left(-\frac{1}{\tau}\right)^{\rho} \int_{s} \boldsymbol{\Phi}_{n-\rho}\left(\mathbf{r}^{\prime}\right) \times \hat{\mathbf{n}^{\prime}}\left(\hat{\mathbf{r}} \cdot \mathbf{r}^{\prime}\right)^{\rho} d S\left(\mathbf{r}^{\prime}\right), \\
\boldsymbol{l}_{p} & =\frac{1}{4 \pi} \sum_{n=0}^{\infty} \frac{(i \tau k)^{n}}{n !} \sum_{\rho=0}^{n}\left(\begin{array}{l}
n \\
\rho
\end{array}\right)(-1)^{\rho} \int_{s} T_{\mathbf{r}^{\prime}} \boldsymbol{\Phi}_{n-\rho}\left(\mathbf{r}^{\prime}\right)\left(\hat{\mathbf{r}} \cdot \mathbf{r}^{\prime}\right)^{\rho} d S\left(\mathbf{r}^{\prime}\right), \\
\boldsymbol{l}_{s} & =\frac{1}{4 \pi} \sum_{n=0}^{\infty} \frac{(i \tau k)^{n}}{n !} \sum_{\rho=0}^{n}\left(\begin{array}{l}
n \\
\rho
\end{array}\right)\left(-\frac{1}{\tau}\right)^{\rho} \int_{s} T_{\mathbf{r}^{\prime}} \boldsymbol{\Phi}_{n-\rho}\left(\mathbf{r}^{\prime}\right)\left(\hat{\mathbf{r}} \cdot \mathbf{r}^{\prime}\right)^{\rho} d S\left(\mathbf{r}^{\prime}\right) .
\end{aligned}
$$

Inserting the expressions (113)-(117) into (56)-(61) it yields

$$
\begin{aligned}
& g_{r}^{\text {rigid }}(\hat{\mathbf{r}}, \hat{\mathbf{k}})=\frac{1}{4 \pi c_{p}^{2}} \sum_{n=0}^{\infty} \frac{(i \tau k)^{n+1}}{n !} \sum_{\rho=0}^{n}\left(\begin{array}{l}
n \\
\rho
\end{array}\right)(-1)^{\rho+1} \hat{\mathbf{r}} \cdot \int_{s} T_{\mathbf{r}^{\prime}} \boldsymbol{\Phi}_{n-\rho}\left(\mathbf{r}^{\prime}\right)\left(\hat{\mathbf{r}} \cdot \mathbf{r}^{\prime}\right)^{\rho} d S\left(\mathbf{r}^{\prime}\right) \\
& g_{\vartheta}^{\text {rigid }}(\hat{\mathbf{r}}, \hat{\mathbf{k}})=\frac{1}{4 \pi c_{s}^{2}} \sum_{n=0}^{\infty} \frac{(i \tau k)^{n+1}}{n !} \sum_{\rho=0}^{n}\left(\begin{array}{l}
n \\
\rho
\end{array}\right)\left(-\frac{1}{\tau}\right)^{\rho+1} \hat{\boldsymbol{\vartheta}} \cdot \int_{s} T_{\mathbf{r}^{\prime}} \boldsymbol{\Phi}_{n-\rho}\left(\mathbf{r}^{\prime}\right)\left(\hat{\mathbf{r}} \cdot \mathbf{r}^{\prime}\right)^{\rho} d S\left(\mathbf{r}^{\prime}\right)
\end{aligned}
$$




$$
g_{\varphi}^{\text {rigid }}(\hat{\mathbf{r}}, \hat{\mathbf{k}})=\frac{1}{4 \pi c_{s}^{2}} \sum_{n=0}^{\infty} \frac{(i \tau k)^{n+1}}{n !} \sum_{\rho=0}^{n}\left(\begin{array}{l}
n \\
\rho
\end{array}\right)\left(-\frac{1}{\tau}\right)^{\rho+1} \hat{\boldsymbol{\varphi}} \cdot \int_{s} T_{\mathbf{r}^{\prime}} \boldsymbol{\Phi}_{n-\rho}\left(\mathbf{r}^{\prime}\right)\left(\hat{\mathbf{r}} \cdot \mathbf{r}^{\prime}\right)^{\rho} d S\left(\mathbf{r}^{\prime}\right),
$$

for the case of a rigid scatterer and

$$
\begin{aligned}
g_{r}^{\text {cavity }}(\hat{\mathbf{r}}, \hat{\mathbf{k}})= & \frac{1}{4 \pi} \sum_{n=1}^{\infty} \frac{(i \tau k)^{n+2}}{n !} \sum_{\rho=0}^{n}\left(\begin{array}{l}
n \\
\rho
\end{array}\right)(-1)^{\rho+1} \\
& \cdot\left[\left(1-2 \tau^{2}\right) \tilde{\mathbf{I}}+2 \tau^{2} \hat{\mathbf{r}} \otimes \hat{\mathbf{r}}\right]: \int_{s} \boldsymbol{\Phi}_{n-\rho}\left(\mathbf{r}^{\prime}\right) \otimes \hat{\mathbf{n}}^{\prime}\left(\hat{\mathbf{r}} \cdot \mathbf{r}^{\prime}\right)^{\rho} d S\left(\mathbf{r}^{\prime}\right), \\
g_{\vartheta}^{\text {cavity }}(\hat{\mathbf{r}}, \hat{\mathbf{k}})= & \frac{1}{4 \pi \tau} \sum_{n=1}^{\infty} \frac{(i \tau k)^{n+2}}{n !} \sum_{\rho=0}^{n}\left(\begin{array}{l}
n \\
\rho
\end{array}\right)\left(-\frac{1}{\tau}\right)^{\rho+1} \\
& \cdot \int_{s}\left[2 \hat{\mathbf{r}} \otimes \hat{\boldsymbol{\vartheta}}: \Phi_{n-\rho}\left(\mathbf{r}^{\prime}\right) \otimes \hat{\mathbf{n}}^{\prime}+\hat{\boldsymbol{\varphi}} \cdot \boldsymbol{\Phi}_{n-\rho}\left(\mathbf{r}^{\prime}\right) \times \hat{\mathbf{n}}^{\prime}\right]\left(\hat{\mathbf{r}} \cdot \mathbf{r}^{\prime}\right)^{\rho} d S\left(\mathbf{r}^{\prime}\right), \\
g_{\varphi}^{\text {cavity }}(\hat{\mathbf{r}}, \hat{\mathbf{k}})= & \frac{1}{4 \pi \tau} \sum_{n=1}^{\infty} \frac{(i \tau k)^{n+2}}{n !} \sum_{\rho=0}^{n}\left(\begin{array}{l}
n \\
\rho
\end{array}\right)\left(-\frac{1}{\tau}\right)^{\rho+1} \\
& \cdot \int_{s}\left[2 \hat{\mathbf{r}} \otimes \hat{\boldsymbol{\varphi}}: \boldsymbol{\Phi}_{n-\rho}\left(\mathbf{r}^{\prime}\right) \otimes \hat{\mathbf{n}}^{\prime}-\hat{\boldsymbol{\vartheta}} \cdot \boldsymbol{\Phi}_{n-\rho}\left(\mathbf{r}^{\prime}\right) \times \hat{\mathbf{n}}^{\prime}\right]\left(\hat{\mathbf{r}} \cdot \mathbf{r}^{\prime}\right)^{\rho} d S\left(\mathbf{r}^{\prime}\right) .
\end{aligned}
$$

The series expansions (121)-(123) start with $n=1$ since all the terms that correspond to $n=0$ involve the surface integral $\int_{s} \hat{\mathbf{n}}^{\prime} d S$ which vanishes by the divergence theorem. Therefore the normalized spherical scattering amplitudes are $O(k)$ for the rigid scatterer and $O\left(k^{3}\right)$ for the cavity, as $k \rightarrow 0$. In particular the leading term approximations for the rigid scatterer, as $k \rightarrow 0$, are

$$
\begin{aligned}
& g_{r}^{\text {rigid }}(\hat{\mathbf{r}}, \hat{\mathbf{k}})=-\frac{i k}{4 \pi \mu} \tau^{3} \hat{\mathbf{r}} \cdot \int_{s} T_{\mathbf{r}^{\prime}} \boldsymbol{\Phi}_{0}\left(\mathbf{r}^{\prime}\right) d S\left(\mathbf{r}^{\prime}\right)+O\left(k^{2}\right), \\
& g_{\vartheta}^{\text {rigid }}(\hat{\mathbf{r}}, \hat{\mathbf{k}})=-\frac{i k}{4 \pi \mu} \hat{\boldsymbol{\vartheta}} \cdot \int_{s} T_{\mathbf{r}^{\prime}} \boldsymbol{\Phi}_{0}\left(\mathbf{r}^{\prime}\right) d S\left(\mathbf{r}^{\prime}\right)+O\left(k^{2}\right), \\
& g_{\varphi}^{\text {rigid }}(\hat{\mathbf{r}}, \hat{\mathbf{k}})=-\frac{i k}{4 \pi \mu} \hat{\varphi} \cdot \int_{s} T_{\mathbf{r}^{\prime}} \Phi_{0}\left(\mathbf{r}^{\prime}\right) d S\left(\mathbf{r}^{\prime}\right)+O\left(k^{2}\right) .
\end{aligned}
$$

Similarly, by means of the formula

$$
\int_{s}^{\hat{\mathbf{n}}^{\prime}} \otimes \mathbf{r}^{\prime} d S\left(\mathbf{r}^{\prime}\right)=\int_{V^{-}} \nabla \mathbf{r}^{\prime} d V=V \tilde{\mathbf{I}},
$$

where $V$ is the volume of the scatterer. It is obtained that the leading term approximations for the case of a cavity, as $k \rightarrow 0$, are

$$
\begin{aligned}
g_{r}^{\text {cavity }}(\hat{\mathbf{r}}, \hat{\mathbf{k}})= & -\frac{i k^{3} \tau^{3}}{4 \pi}\left[\left(1-2 \tau^{2}\right) \tilde{\mathbf{I}}+2 \tau^{2} \hat{\mathbf{r}} \otimes \hat{\mathbf{r}}\right] \\
& \cdot\left[V \mathbf{a}_{0} \otimes \hat{\mathbf{r}}-\int_{s} \boldsymbol{\Phi}_{1}\left(\mathbf{r}^{\prime}\right) \otimes \hat{\mathbf{n}}^{\prime} d S\left(\mathbf{r}^{\prime}\right)\right]+O\left(k^{4}\right),
\end{aligned}
$$




$$
\begin{aligned}
& g_{\vartheta}^{\text {cavity }}(\hat{\mathbf{r}}, \hat{\mathbf{k}})=-\frac{i k^{3} \tau}{4 \pi}\left[\frac{V}{\tau} \mathbf{a}_{0} \cdot \hat{\boldsymbol{\vartheta}}-2 \hat{\mathbf{r}} \otimes \hat{\boldsymbol{\vartheta}}: \int_{S} \boldsymbol{\Phi}_{1}\left(\mathbf{r}^{\prime}\right) \otimes \mathbf{n}^{\prime} d S\left(\mathbf{r}^{\prime}\right)\right. \\
& \left.-\hat{\boldsymbol{\varphi}} \cdot \int_{s} \boldsymbol{\Phi}_{1}\left(\mathbf{r}^{\prime}\right) \times \hat{\mathbf{n}}^{\prime} d S\left(\mathbf{r}^{\prime}\right)\right]+O\left(k^{4}\right), \\
& g_{\varphi}^{\text {cavity }}(\hat{\mathbf{r}}, \hat{\mathbf{k}})=-\frac{i k^{3} \tau}{4 \pi}\left[\frac{V}{\tau} \mathbf{a}_{0} \cdot \hat{\varphi}-2 \hat{\mathbf{r}} \otimes \hat{\boldsymbol{\varphi}}: \int_{s} \boldsymbol{\Phi}_{1}\left(\mathbf{r}^{\prime}\right) \otimes \hat{\mathbf{n}}^{\prime} d S\left(\mathbf{r}^{\prime}\right)\right. \\
& \left.+\hat{\boldsymbol{\vartheta}} \cdot \int_{s} \boldsymbol{\Phi}_{1}\left(\mathbf{r}^{\prime}\right) \times \hat{\mathbf{n}}^{\prime} d S\left(\mathbf{r}^{\prime}\right)\right]+O\left(k^{4}\right) .
\end{aligned}
$$

The low-frequency expansions for the scattering cross-sections are derived by substituting the corresponding expansions (118)-(123) into the formulae (70) and (71). The leading term approximations, as $k \rightarrow 0$, for the four problems of concern here, can be obtained by first expressing the relative integrals in terms of $\hat{\mathbf{r}}$ alone, then use formulae [see Appendix].

$$
\begin{gathered}
\int_{\mid \hat{\mathbf{r}}=1} \hat{\mathbf{r}} d \Omega(\hat{\mathbf{r}})=\mathbf{0}, \\
\int_{\mid \hat{\mathbf{r}}=1} \hat{\mathbf{r}} \otimes \hat{\mathbf{r}} d \Omega(\hat{\mathbf{r}})=\frac{4 \pi}{3} \tilde{\mathbf{I}}, \\
\int_{|\hat{\mathbf{r}}|=1} \hat{\mathbf{r}} \otimes \hat{\mathbf{r}} \otimes \hat{\mathbf{r}} d \Omega(\hat{\mathbf{r}})=\mathbf{0} \otimes \mathbf{0} \otimes \mathbf{0}, \\
\int_{\mid \hat{\mathbf{r}}=1} \hat{\mathbf{r}} \otimes \hat{\mathbf{r}} \otimes \hat{\mathbf{r}} \otimes \hat{\mathbf{r}} d \Omega(\hat{\mathbf{r}}) \\
=\frac{4 \pi}{15}\left[\tilde{\mathbf{I}} \otimes \tilde{\mathbf{I}}+\sum_{i, j=1}^{3} \hat{\mathbf{e}}_{i} \otimes \hat{\mathbf{e}}_{j} \otimes \hat{\mathbf{e}}_{i} \otimes \hat{\mathbf{e}}_{j}+\sum_{i=1}^{3} \hat{\mathbf{e}}_{i} \otimes \tilde{\mathbf{I}} \otimes \hat{\mathbf{e}}_{i}\right]
\end{gathered}
$$

and finally perform the indicated contractions.

Following this program one obtains for

(i) $P$-incidence on a rigid scatterer.

$$
\begin{aligned}
\sigma_{\text {rigid }}^{p}= & \frac{\tau}{16 \pi^{2} \mu^{2}} \int_{|\hat{\mathbf{r}}|=1}\left[\tau^{3}\left|\hat{\mathbf{r}} \cdot \int_{s} T \boldsymbol{\Phi}_{0} d S\left(\mathbf{r}^{\prime}\right)\right|^{2}\right. \\
& \left.\quad+\left|\hat{\boldsymbol{\vartheta}} \cdot \int_{s} T \boldsymbol{\Phi}_{0} d S\left(\mathbf{r}^{\prime}\right)\right|^{2}+\left|\hat{\boldsymbol{\varphi}} \cdot \int_{s} T \boldsymbol{\Phi}_{0} d S\left(\mathbf{r}^{\prime}\right)\right|^{2}\right] d \Omega(\hat{\mathbf{r}})+O\left(k^{2}\right) \\
= & \frac{\tau}{16 \pi^{2} \mu^{2}} \int_{s} T \boldsymbol{\Phi}_{0} d S\left(\mathbf{r}^{\prime}\right) \cdot \int_{\mid \hat{\mathbf{r}}=1}\left[\tilde{\mathbf{I}}+\left(\tau^{3}-1\right) \hat{\mathbf{r}} \otimes \hat{\mathbf{r}}\right] d \Omega(\hat{\mathbf{r}}) \cdot \int_{S} T \boldsymbol{\Phi}_{0} d S\left(\mathbf{r}^{\prime}\right)+O\left(k^{2}\right) \\
= & \frac{\tau\left(\tau^{3}+2\right)}{12 \pi \mu^{2}}\left|\int_{s} T \boldsymbol{\Phi}_{0} d S\left(\mathbf{r}^{\prime}\right)\right|^{2}+O\left(k^{2}\right) .
\end{aligned}
$$


(ii) $S$-incidence on a rigid scatterer.

$$
\sigma_{\text {rigid }}^{s}=\frac{1}{\tau}\left[\text { the same as } \sigma_{\text {rigid }}^{p}, \text { with } \boldsymbol{\Phi}_{0} \text { corresponding to } S \text {-incidence }\right] .
$$

(iii) $P$-incidence on a cavity.

$$
\begin{aligned}
& \boldsymbol{\sigma}_{\text {cavity }}^{p}=\frac{\tau^{3} k^{4}}{16 \pi^{2}} \int_{\mid \hat{\mid}=1}\left\{\tau\left|\left[\left(1-2 \tau^{2}\right) \tilde{\mathbf{I}}+2 \tau^{2} \hat{\mathbf{r}} \otimes \hat{\mathbf{r}}\right]:\left[V \mathbf{a}_{0} \otimes \hat{\mathbf{r}}-\int_{s} \boldsymbol{\Phi}_{1}\left(\mathbf{r}^{\prime}\right) \otimes \hat{\mathbf{n}}^{\prime} d S\left(\mathbf{r}^{\prime}\right)\right]\right|^{2}\right. \\
& +\left|\frac{V}{\tau} \mathbf{a}_{0} \cdot \hat{\boldsymbol{\vartheta}}-2 \hat{\mathbf{r}} \otimes \hat{\boldsymbol{\vartheta}}: \int_{S} \boldsymbol{\Phi}_{1}\left(r^{\prime}\right) \otimes \hat{\mathbf{n}}^{\prime} d S\left(\mathbf{r}^{\prime}\right)-\hat{\boldsymbol{\varphi}} \cdot \int_{S} \boldsymbol{\Phi}_{1}\left(\mathbf{r}^{\prime}\right) \times \hat{\mathbf{n}}^{\prime} d S\left(\mathbf{r}^{\prime}\right)\right|^{2} \\
& \left.+\left|\frac{V}{\tau} \mathbf{a}_{0} \cdot \hat{\boldsymbol{\varphi}}-2 \hat{\mathbf{r}} \otimes \hat{\boldsymbol{\varphi}}: \int_{S} \boldsymbol{\Phi}_{1}\left(\mathbf{r}^{\prime}\right) \otimes \hat{\mathbf{n}}^{\prime} d S\left(\mathbf{r}^{\prime}\right)+\hat{\boldsymbol{\vartheta}} \cdot \int_{S} \boldsymbol{\Phi}_{1}\left(\mathbf{r}^{\prime}\right) \times \hat{\mathbf{n}}^{\prime} d S\left(r^{\prime}\right)\right|^{2}\right\} d \Omega(\hat{\mathbf{r}}) \\
& +O\left(k^{6}\right) \\
& =\frac{\tau^{3} k^{4}}{16 \pi^{2}}\left\{\frac{8 \pi V^{2}}{3 \tau^{2}}+\tau V^{2} \mathbf{a}_{0} \cdot \int_{\mid \hat{\boldsymbol{r}}=1} \hat{\mathbf{r}} \otimes \hat{\mathbf{r}} d \Omega(\hat{\mathbf{r}}) \cdot \mathbf{a}_{0}\right. \\
& +\int_{s} \boldsymbol{\Phi}_{1}\left(\mathbf{r}^{\prime}\right) \otimes \hat{\mathbf{n}}^{\prime} d S\left(\mathbf{r}^{\prime}\right): \int_{\mid \hat{\mid}=1}\left[\tau\left(1-2 \tau^{2}\right)^{2} \tilde{\mathbf{I}} \otimes \tilde{\mathbf{I}}+2 \tau^{3}\left(1-2 \tau^{2}\right)(\tilde{\mathbf{I}} \otimes \mathbf{r} \otimes \hat{\mathbf{r}}+\hat{\mathbf{r}} \otimes \hat{\mathbf{r}} \otimes \tilde{\mathbf{I}})\right. \\
& \left.+4 \hat{\mathbf{r}} \otimes \tilde{\mathbf{I}} \otimes \hat{\mathbf{r}}+4\left(\tau^{5}-1\right) \hat{\mathbf{r}} \otimes \hat{\mathbf{r}} \otimes \hat{\mathbf{r}} \otimes \hat{\mathbf{r}}\right] d \Omega(\hat{\mathbf{r}}) \\
& : \int_{s}^{\hat{\mathbf{n}}^{\prime}} \otimes \boldsymbol{\Phi}_{1}\left(\mathbf{r}^{\prime}\right) d S\left(\mathbf{r}^{\prime}\right)+\int_{s} \boldsymbol{\Phi}_{1}\left(\mathbf{r}^{\prime}\right) \times \hat{\mathbf{n}}^{\prime} d S\left(\mathbf{r}^{\prime}\right) \cdot \int_{|\hat{\mid}|=1}(\tilde{\mathbf{I}}-\hat{\mathbf{r}} \otimes \hat{\mathbf{r}}) d \Omega(\hat{\mathbf{r}}) \cdot \int_{S} \boldsymbol{\Phi}_{1}\left(\mathbf{r}^{\prime}\right) \times \hat{\mathbf{n}}^{\prime} d S\left(\mathbf{r}^{\prime}\right) \\
& \left.-4 \int_{S} \boldsymbol{\Phi}_{1}\left(\mathbf{r}^{\prime}\right) \otimes \hat{\mathbf{n}}^{\prime} d S\left(\mathbf{r}^{\prime}\right): \int_{\hat{\mid} \mid=1} \hat{\mathbf{r}} \otimes \tilde{\mathbf{I}} \times \hat{\mathbf{r}} d \Omega(\hat{\mathbf{r}}) \cdot \int_{S} \boldsymbol{\Phi}_{1}\left(r^{\prime}\right) \times \hat{\mathbf{n}}^{\prime} d S\left(\mathbf{r}^{\prime}\right)\right\}+O\left(k^{6}\right) \\
& =\frac{\tau^{3} k^{4}}{60 \pi}\left[\frac{5\left(\tau^{3}+2\right)}{\tau^{2}} V^{2}+\left(28 \tau^{5}-40 \tau^{3}+15 \tau-8\right)\left|\int_{S} \boldsymbol{\Phi}_{1}\left(\mathbf{r}^{\prime}\right) \cdot \hat{\mathbf{n}}^{\prime} d S\left(\mathbf{r}^{\prime}\right)\right|^{2}\right. \\
& \left.-10\left|\int_{S} \boldsymbol{\Phi}_{1}\left(\mathbf{r}^{\prime}\right) \times \hat{\mathbf{n}}^{\prime} d S\left(\mathbf{r}^{\prime}\right)\right|^{2}+4\left(\tau^{5}+4\right)\left\|\int_{S} \boldsymbol{\Phi}_{1}\left(\mathbf{r}^{\prime}\right) \otimes \hat{\mathbf{n}}^{\prime} d S\left(\mathbf{r}^{\prime}\right)\right\|^{2}\right]+O\left(k^{6}\right),
\end{aligned}
$$

where the norm of a dyadic is defined as

$$
\|\mathbf{a} \otimes \mathbf{b}\|^{2}=\sum_{i, j=1}^{3}\left(a_{i} b_{i}\right)^{2} .
$$

In the third part of (137) all terms that evaluate to zero has been omitted. 
(iv) $S$-incidence on a cavity.

$$
\sigma_{\text {cavity }}^{s}=\frac{1}{\tau}\left[\text { the same as } \sigma_{\text {cavity }}^{p} \text {, with } \boldsymbol{\Phi}_{1} \text { corresponding to } S \text {-incidence }\right] \text {. }
$$

From (135) and (137) it is observed that the leading low-frequency approximation of the scattering cross-section for the rigid scatterer is independent of the wave number, while for the cavity the corresponding approximation is of the order of $k^{4}$.

In other words, the total energy scattered by a rigid scatterer is by four orders of magnitude of the wave number, more than the corresponding total energy scattered by a cavity. Comparing the above results with Rayleigh's law of scattering for sound waves, which says that in the low-frequency limit the total energy scattered by a rigid scatterer is proportional to the fourth inverse power of the wavelength, it is observed that in the case of elastic waves Rayleigh's law holds true for the case of a cavity. The corresponding energy scattered by a rigid body is independent of the wavelength. Comparing the results (135) and (137) with the corresponding results (73), (74) of Barratt and Collins [2] it is observed that the leading term approximation, as $k \rightarrow 0$, of the scattering cross-section using the expressions (73), (74) demand the knowledge of the coefficients $\boldsymbol{\Phi}_{0}$, $\boldsymbol{\Phi}_{1}$ for the rigid scatterer and the coefficients $\Phi_{0}, \Phi_{1}, \Phi_{2}, \Phi_{3}, \Phi_{4}$ for the cavity while by means of (135) and (137) the corresponding approximations are obtained when it is known only $\boldsymbol{\Phi}_{0}$ for the rigid scatterer and $\boldsymbol{\Phi}_{0}, \boldsymbol{\Phi}_{1}$ for the cavity. Considering the rapidly increasing difficulty in evaluating the coefficients $\boldsymbol{\Phi}_{n}$ for specific problems [7, 8], this technique of evaluating the scattering cross-section using the lowest possible coefficients is very efficient.

Appendix. The divergence theorem implies that

$$
\int_{\mid \hat{\mathbf{r}}=1} \hat{\mathbf{r}} d \Omega(\hat{\mathbf{r}})=\int_{|\boldsymbol{r}| \leq 1}(\nabla 1) d V(\mathbf{r})=\mathbf{0} .
$$

Alone the same line, it is obtained that

$$
\begin{aligned}
\int_{|\hat{\mathbf{r}}|=1} \hat{\mathbf{r}} \otimes \hat{\mathbf{r}} d \Omega(\hat{\mathbf{r}}) & =\int_{|\mathbf{r}| \leqslant 1} \nabla \hat{\mathbf{r}} d V(\mathbf{r})=\int_{|\mathbf{r}| \leqslant 1} V\left(\frac{\mathbf{r}}{r}\right) d V(\mathbf{r}) \\
& =\int_{0}^{1} \int_{|\hat{\mathbf{r}}|=1}\left(-\frac{\hat{\mathbf{r}} \otimes \hat{\mathbf{r}}}{r}+\frac{\tilde{\mathbf{I}}}{r}\right) r^{2} d \Omega(\hat{\mathbf{r}}) d r=-\frac{1}{2} \int_{|\hat{\mathbf{r}}|=1} \hat{\mathbf{r}} \otimes \hat{\mathbf{r}} d \Omega(\hat{\mathbf{r}})+\frac{4 \pi}{2} \tilde{\mathbf{I}}
\end{aligned}
$$

and by solving with respect to the surface integral it is implied that

$$
\int_{\mid \hat{\mathbf{r}}=1} \hat{\mathbf{r}} \otimes \hat{\mathbf{r}} d \Omega(\hat{\mathbf{r}})=\frac{4 \pi}{3} \tilde{\mathbf{I}} .
$$

Formula (A.1) denotes that the 3 integrals over the unit sphere of all first degree monomials of the directional cosines of $\hat{\mathbf{r}}$ are zero.

Similarly, Formula (A.3) denotes that among the $3^{2}=9$ corresponding integrals of second degree monomials there are only three different to zero. They correspond to the base dyads $\hat{\mathbf{e}}_{i} \otimes \hat{\mathbf{e}}_{i}, i=1,2,3$ and they all have the same value $4 \pi / 3$. For the $3^{3}=27$ 
integrals of the third degree monomials of the directional cosines, the divergence theorem provides

$$
\begin{aligned}
\int_{\hat{\mathbf{r}}=1} \hat{\mathbf{r}} \otimes \hat{\mathbf{r}} \otimes \hat{\mathbf{r}} d \Omega(\hat{\mathbf{r}}) & =\int_{|\mathbf{r}| \leq 1} \nabla \hat{\mathbf{r}} \otimes \hat{\mathbf{r}} d V(\mathbf{r})=\int_{|\mathbf{r}| \leq 1} \nabla\left(\frac{\mathbf{r} \otimes \mathbf{r}}{r^{2}}\right) d V(\mathbf{r}) \\
& =\int_{0}^{1} \int_{\mid \hat{|r|}=1}\left(\frac{-2 \hat{\mathbf{r}} \otimes \hat{\mathbf{r}} \otimes \hat{\mathbf{r}}+\tilde{\mathbf{I}} \otimes \hat{\mathbf{r}}+\hat{\mathbf{r}} \otimes \tilde{\mathbf{I}}}{r}\right) r^{2} d \Omega(\hat{\mathbf{r}}) d r \\
& =-\int_{\mid \hat{\mathbf{r}}=1} \hat{\mathbf{r}} \otimes \hat{\mathbf{r}} \otimes \hat{\mathbf{r}} d \Omega(\hat{\mathbf{r}})+\frac{1}{2} \int_{\mid \hat{\mathbf{r}}=1}(\tilde{\mathbf{I}} \otimes \hat{\mathbf{r}}+\hat{\mathbf{r}} \otimes \tilde{\mathbf{I}}) d \Omega(\hat{\mathbf{r}}) .
\end{aligned}
$$

From (A.1) it is concluded that

$$
\begin{aligned}
\int_{|\hat{\mathbf{r}}|=1} \hat{\mathbf{r}} \otimes \hat{\mathbf{r}} \otimes \hat{\mathbf{r}} d \Omega(\hat{\mathbf{r}}) & =\frac{1}{4} \tilde{\mathbf{I}} \otimes \int_{|\hat{\mathbf{r}}|=1} \hat{\mathbf{r}} d \Omega(\hat{\mathbf{r}})+\frac{1}{4} \int_{\mid \hat{|r|}=1} \hat{\mathbf{r}} d \Omega(\hat{\mathbf{r}}) \otimes \tilde{\mathbf{I}} \\
& =\mathbf{0} \otimes \mathbf{0} \otimes \mathbf{0} .
\end{aligned}
$$

Therefore all the third degree monomials of the directional cosines of $\hat{\mathbf{r}}$ have zero integrals over the unit sphere. For the $3^{4}=81$ integrals of the fourth degree monomials it follows that

$$
\begin{aligned}
\int_{\mid \hat{|r|}=1} \hat{\mathbf{r}} \otimes \hat{\mathbf{r}} \otimes \hat{\mathbf{r}} \otimes \hat{\mathbf{r}} d \Omega(\hat{\mathbf{r}})=\int_{|\mathbf{r}| \leqslant 1} \nabla \hat{\mathbf{r}} \otimes \hat{\mathbf{r}} \otimes \hat{\mathbf{r}} d \Omega(\hat{\mathbf{r}}) \\
=\int_{\mid \mathbf{r} \leqslant 1} \nabla\left(\frac{\mathbf{r} \otimes \mathbf{r} \otimes \mathbf{r}}{r^{3}}\right) d V(\mathbf{r}) \\
=\int_{0}^{1} \int_{\mid \hat{|r|}=1} \frac{-3 \hat{\mathbf{r}} \otimes \hat{\mathbf{r}} \otimes \hat{\mathbf{r}} \otimes \hat{\mathbf{r}}+\tilde{\mathbf{I}} \otimes \hat{\mathbf{r}} \otimes \hat{\mathbf{r}}+\hat{\mathbf{r}} \otimes \tilde{\mathbf{I}} \otimes \hat{\mathbf{r}}+\hat{\mathbf{r}} \otimes \hat{\mathbf{r}} \otimes \tilde{\mathbf{I}}}{r} r^{2} d \Omega(\hat{\mathbf{r}}) d r \\
=-\frac{3}{2} \int_{\mid \hat{\mathbf{r}}=1} \hat{\mathbf{r}} \otimes \hat{\mathbf{r}} \otimes \hat{\mathbf{r}} \otimes \hat{\mathbf{r}} d \Omega(\hat{\mathbf{r}}) \\
\quad+\frac{1}{2} \int_{\mid \hat{r}=1}[\tilde{\mathbf{I}} \otimes \hat{\mathbf{r}} \otimes \hat{\mathbf{r}}+\hat{\mathbf{r}} \otimes \tilde{\mathbf{I}} \otimes \hat{\mathbf{r}}+\hat{\mathbf{r}} \otimes \hat{\mathbf{r}} \otimes \tilde{\mathbf{I}}] d \Omega(\hat{\mathbf{r}}) .
\end{aligned}
$$

Inserting (A.3) into (A.6) it is confirmed that

$$
\begin{aligned}
\int_{\mid \hat{i}=1} \hat{\mathbf{r}} \otimes \hat{\mathbf{r}} \otimes \hat{\mathbf{r}} \otimes \hat{\mathbf{r}} d \Omega(\hat{\mathbf{r}}) & =\frac{4 \pi}{15}\left[\hat{\mathbf{e}}_{i} \otimes \hat{\mathbf{e}}_{i} \otimes \hat{\mathbf{e}}_{j} \otimes \hat{\mathbf{e}}_{j}+\hat{\mathbf{e}}_{i} \otimes \hat{\mathbf{e}}_{j} \otimes \hat{\mathbf{e}}_{i} \otimes \hat{\mathbf{e}}_{j}+\hat{\mathbf{e}}_{i} \otimes \hat{\mathbf{e}}_{j} \otimes \hat{\mathbf{e}}_{j} \otimes \hat{\mathbf{e}}_{i}\right] \\
& =\frac{4 \pi}{15}\left[\tilde{\mathbf{I}} \otimes \tilde{\mathbf{I}}+\hat{\mathbf{e}}_{i} \otimes \hat{\mathbf{e}}_{j} \otimes \hat{\mathbf{e}}_{i} \otimes \hat{\mathbf{e}}_{j}+\hat{\mathbf{e}}_{i} \otimes \tilde{\mathbf{I}} \otimes \hat{\mathbf{e}}_{i}\right], \quad \text { (A.7) }
\end{aligned}
$$

where repeated indices, in (A.7), indicate summation from 1 to 3 . Continuing this technique for higher degree monomials it is concluded that the integrals over the unit sphere of all odd degree monomials of the directional cosines of $\hat{\mathbf{r}}$ are zero. In addition, those of the integrals of the above monomials, of degree $2 n$, that, do not vanish, have the same value which is equal to $4 \pi /(2 n+1)$ !!, where the double factorial represents the product of the odd natural numbers from 1 to $(2 n+1)$. 
The above computational technique is very efficient, especially where the degree of the monomials is $n>2$, e.g. for $n=8$ there are $3^{8}=6.561$ integrals to evaluate, where with the use of the above method there is no need to evaluate any integral.

\section{REFERENCES}

[1] R. P. Banaugh, Application of integral representations of displacement potentials in elasto-dynamics, Bull. Seism. Soc. Amer. 54, 1073 (1964)

[2] P. J. Barratt and W. D. Collins, The scattering cross-section of an obstacle in an elastic solid for plane harmonic waves, Proc. Camb. Philos. Soc. 61, 969 (1965)

[3] G. Dassios, Convergent low-frequency expansions for penetrable scatterers, J. Math. Phys. 18, 126 (1977)

[4] __ Second order low-frequency scattering by the soft ellipsoid, SIAM J. Appl. Math. 38, 373 (1980)

[5] __ Scattering of acoustic waves by a coated pressure-release ellipsoid, J. Acoust. Soc. Amer. 70, 176 (1981)

[6] Low-frequency scattering theory for a penetrable body with an impenetrable core, SIAM J. Appl. Math. 42, 272 (1982)

[7] G. Dassios and K. Kiriaki, The rigid ellipsoid in the presence of a low-frequency elastic wave (to be published)

[8] __ The ellipsoidal cavity in the presence of a low-frequency elastic wave (to be published)

[9] N. G. Einspruch, E. J. Witterholt and R. Truell, Scattering of a plane transverse wave by a spherical obstacle in an elastic medium, J. Appl. Phys. 31, 806 (1960)

[10] V. D. Kupradze, Progress in solid mechanics. III, Dynamical problems in elasticity, North-Holland (1963)

[11] E. G. Lawrence, Diffraction of elastic waves by a rigid inclusion, Quart. J. Mech. Appl. Math. XXIII, 389 (1970)

[12] _ Diffraction of elastic waves by a rigid ellipsoid, Quart. J. Mech. Appl. Math. XXV, 161 (1972)

[13] Y. H. Pao and Varatharajulu, Huygens' principle, radiation conditions, and integral formulas for the scattering of elastic waves, J. Acoust. Soc. Amer. 59, 1361 (1976)

[14] V. Twersky, Certain transmission and reflection theorems, J. Appl. Phys. 25, 859 (1954)

[15] __ Rayleigh scattering, Appl. Opt. 3, 1150 (1964)

[16] P. C. Waterman, Matrix theory of elastic wave scattering, J. Acoust. Soc. Amer. 60, 567 (1976)

[17] __ Matrix theory of elastic wave scattering. II, A new conservation law, J. Acoust. Soc. Amer. 63, 1320 (1978)

[18] L. T. Wheeler and E. Sternberg, Some theorems in classical elastodynamics, Arch. Rat. Mech. Anal. 31, 51 (1968)

[19] C. F. Ying and R. Truell, Scattering of a plane longitudinal wave by a spherical obstacle in an isotropically elastic solid, J. Appl. Phys. 27, 1086 (1956) 